\title{
Energy Frontier Research Center, Center for Materials Science of Nuclear Fuels
}

\author{
Todd R. Allen
}

April 2011

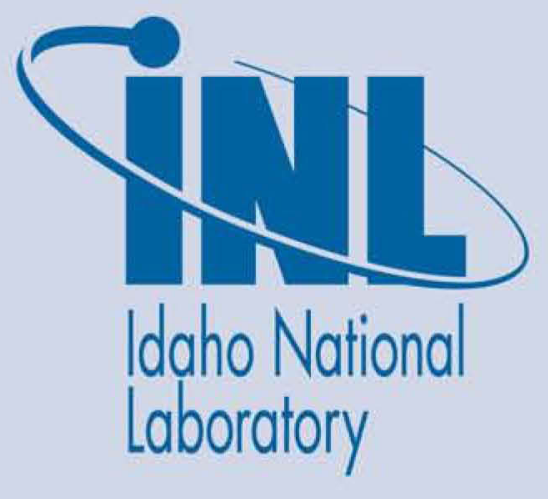

The INL is a U.S. Department of Energy National Laboratory operated by Battelle Energy Alliance 


\section{DISCLAIMER}

This information was prepared as an account of work sponsored by an agency of the U.S. Government. Neither the U.S. Government nor any agency thereof, nor any of their employees, makes any warranty, expressed or implied, or assumes any legal liability or responsibility for the accuracy, completeness, or usefulness, of any information, apparatus, product, or process disclosed, or represents that its use would not infringe privately owned rights. References herein to any specific commercial product, process, or service by trade name, trade mark, manufacturer, or otherwise, does not necessarily constitute or imply its endorsement, recommendation, or favoring by the U.S. Government or any agency thereof. The views and opinions of authors expressed herein do not necessarily state or reflect those of the U.S. Government or any agency thereof. 


\section{Annual Report}

\section{EFRC Director: Professor Todd Allen}

\section{Participating Institutions and Lead Investigators:}

Argonne National Laboratory (Dr. Dieter Wolf)

Colorado School of Mines (Professor John Moore)

Florida State University (Professor Anter El-Azab)

Idaho National Laboratory (Dr. Dave Hurley, Dr. Jian Gan)

Oak Ridge National Laboratory (Dr. Ben Larson, Dr. Judy Pang, Dr. Gene Ice) University of Florida (Professor Simon Phillpot and Professor Michele Manuel) University of Wisconsin (Professor Todd Allen)

\section{April 29, 2011 \\ Idaho National Laboratory \\ Center for Materials and Science of Nuclear Fuel \\ Idaho Falls, Idaho 83415}

http://www.inl.gov

Prepared for the

U.S. Department of Energy

Office of Science

Under DOE Idaho Operations Office

Contract DE-AC07-05ID14517 



\section{OVERVIEW AND EXECUTIVE SUMMARY}

The aim of the Center for Material Science of Nuclear Fuels (CMSNF) is to establish the foundation for predictive understanding of the effects of irradiation-induced defects on thermal transport in oxide nuclear fuels.

The science driver of the center's investigation is to understand how complex defect and microstructures affect phonon mediated thermal transport in UO2, and achieve this understanding for the particular case of irradiation-induced defects and microstructures. The center's research includes modeling and measurement of thermal transport in oxide fuels with different levels of impurities, lattice disorder, and irradiation-induced microstructure, as well as theoretical and experimental investigation of the evolution of disorder, stoichiometry, and microstructure in nuclear fuel under irradiation. With the premise that thermal transport in irradiated $\mathrm{UO} 2$ is a phonon-mediated energy transport process in a crystalline material with defects and microstructure, a step-by-step approach will be utilized to understand the effects of types of defects and microstructures on the collective phonon dynamics in irradiated UO2.

The microstructure science under irradiation theme, guided by Anter El-Azab (Florida State University) and Todd Allen (University of Wisconsin-Madison), is developing an experimentally validated, atomistically informed mesoscale model to predict microstructure evolution under irradiation. Closely coordinated with these multi-scale are the experimental characterization, on commensurate length scales, of radiation damage and microstructure evolution on samples produced under carefully controlled synthesis conditions.

The theme on thermal transport, guided by David Hurley (Idaho National Laboratory) and Simon Phillpot (University of Florida) builds on the underlying microstructure to predict the thermal conductivity under irradiation. Efforts under this thrust dovetail with modeling efforts involving atomistic simulation of phonon transport and prediction of lattice thermal conductivity using the Boltzmann transport framework.

The research goals of the center are:

- Achieve first-principles based understanding of the impact of complex defect structures on thermal transport in irradiated oxide fuel.

- Achieve the above for the case of irradiation induced defects in oxide fuel. As such, the center will also achieve a first-principles based understanding of the effects of irradiation on stoichiometry and microstructure in oxide fuel.

- Establish a new research direction that integrates the physics of thermal transport and the physics of defect and microstructure in irradiated oxide fuel.

The center will accomplish the above goals by aligning its research efforts in the following directions:

- Modeling the irradiation driven defect processes and microstructure and stoichiometry changes in irradiated oxide fuel and checking the models experimentally (Research Theme 1: Microstructure Science).

- Modeling the physics of thermal transport in defective (irradiated) fuel and checking the models experimentally (Research Theme 2: Thermal Transport).

- Integrating the above two themes to ultimately understand the effect of irradiation on thermal transport in oxide fuel

The center has experienced some key successes over the past year. Some 2010 accomplishments include: 
- Completed the initial phase of phonon dispersion, group velocity and line width measurements at the HFIR reactor using $\mathrm{UO}_{2}$ single crystal sample loaned by Atomic Energy Canada Limited (AECL)

- Characterized the influence of grain boundaries on phonon mediated thermal transport in $\mathrm{CeO}_{2}$ thin films, a surrogate materials for $\mathrm{UO}_{2}$

- Completed an analysis of interatomic potential models for $\mathrm{UO}_{2}$ potentials in the context of thermal conductivity predictions

- Established a Monte Carlo solver for Boltzmann transport equation for phonons; now being tested on $\mathrm{UO}_{2}$.

- Modeled the local off-stoichiometry variation in $\mathrm{UO}_{2}$ as a result of irradiation; a key requisite modeling thermal transport.

- Performed synthesis of $\mathrm{CeO}_{2}$ thin film for thermal transport measurements, and characterized the chemistry and structure of these films by various techniques, including atom probe.

- Conducted ion irradiation of $\mathrm{CeO}_{2}$ samples and performed structural characterization of defects.

- Completed the phase field model for void nucleation and growth under irradiation, a key step towards achieving the same for $\mathrm{UO}_{2}$. 


\section{CONTENTS}

OVERVIEW AND EXECUTIVE SUMMARY ….................................................................................ii

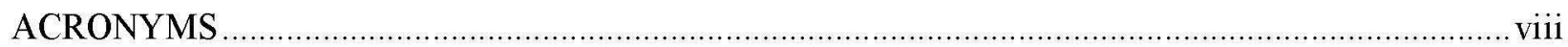

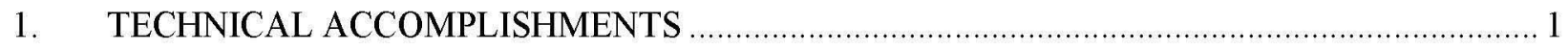

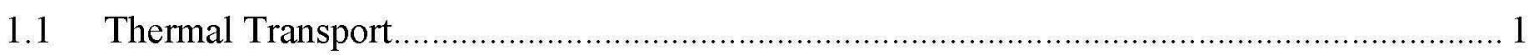

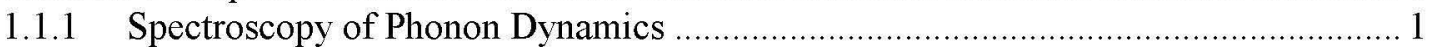

1.1.2 Thermal Conductivity Measurements ...................................................................... 3

1.1.3 Atomistic Simulation of Thermal Transport Properties ........................................... 4

1.1.4 Monte Carlo Solution of the Boltzmann Transport Equation ................................... 6

1.2 Microstructure under Radiation Coupled to Thermal Transport.......................................... 8

1.2.1 Preparation of Thin-Film Samples for Irradiation and Thermal Transport

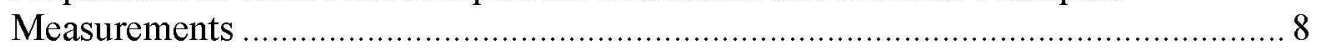

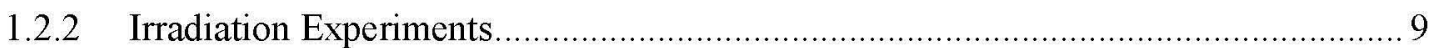

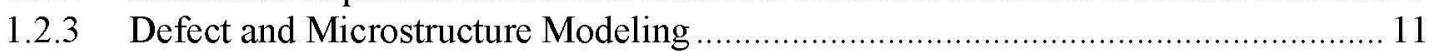

1.2.4 Atom Probe Characterization .................................................................... 12

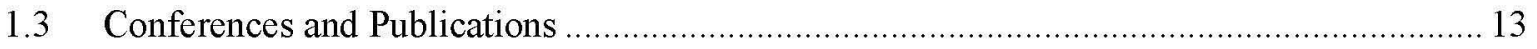

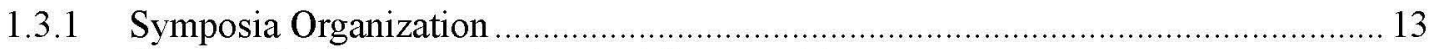

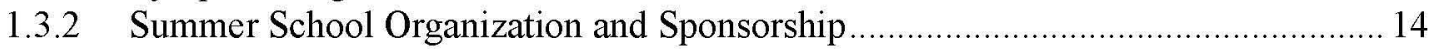

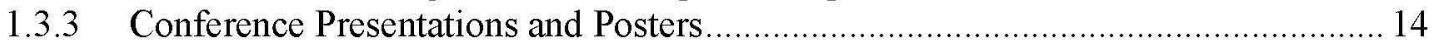

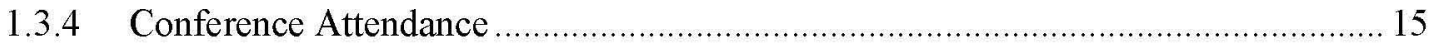

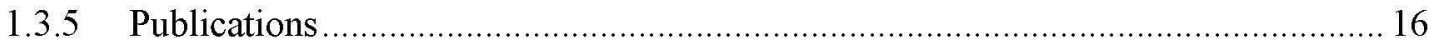

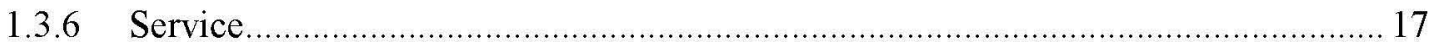

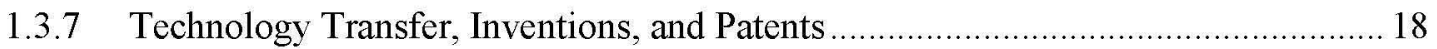

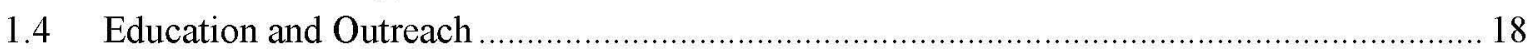

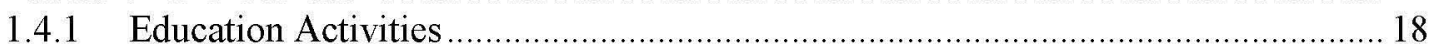

1.4.2 Assessment and Evaluation of Education Activities............................................... 18

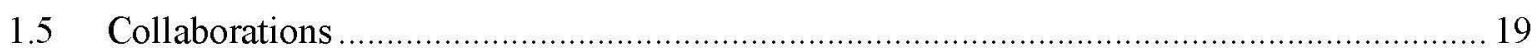

1.5.1 Joint CMSNF-ANL Effort on Computational Microstructure Simulation ............... 19

1.5.2 Scientific Discovery through Advanced Computing Program (SciDAC)................ 19

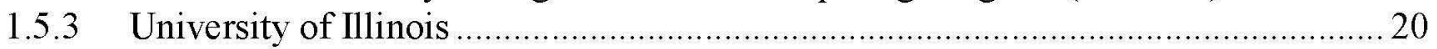

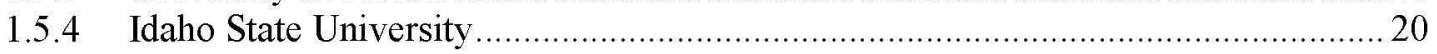

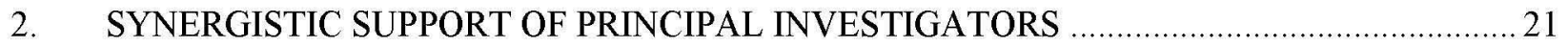

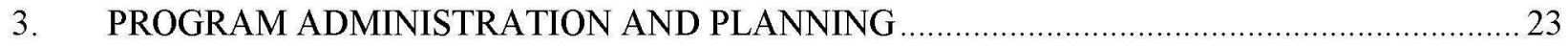

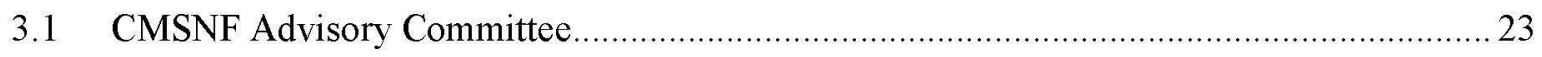

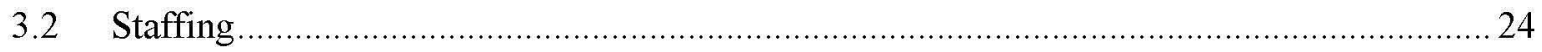

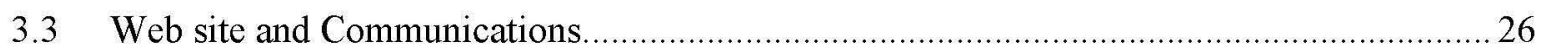

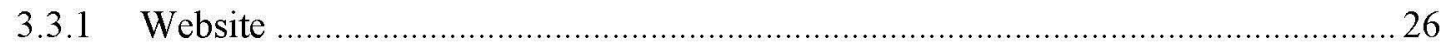

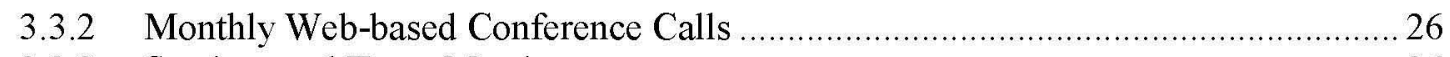

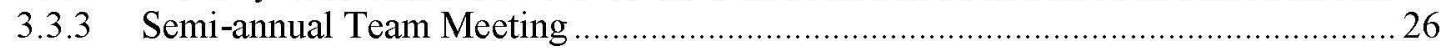

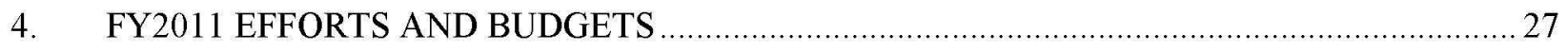

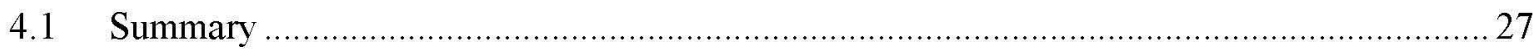

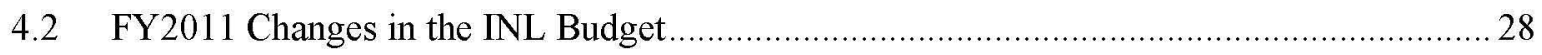




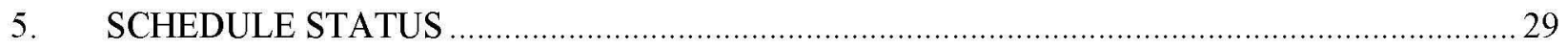

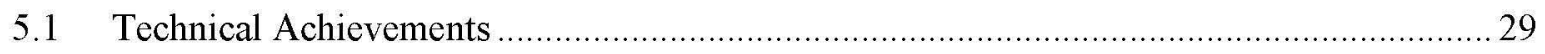

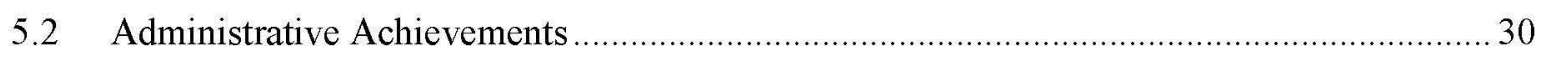

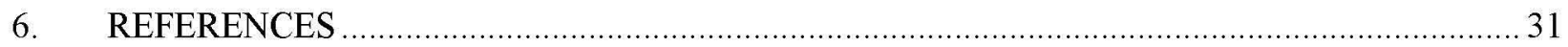

\section{FIGURES}

Figure 1. Longitudinal acoustic phonons in $\mathrm{UO}_{2}$ measured for temperatures of $295 \mathrm{~K}$ and $1200 \mathrm{~K}$; the solid lines are fits to the data.

Figure 2. Measured dispersion curves for $\mathrm{UO}_{2}$ at $295 \mathrm{~K}$ and $1200 \mathrm{~K}$. Circles (triangles) represent transverse (longitudinal) phonons. Blue (red) symbols denote measurements at $295 \mathrm{~K}$ $(1200 \mathrm{~K})$. The lines are guides to the eye and the highlighted line denotes the highvelocity longitudinal optical phonon branch.

Figure 3. Line widths for longitudinal optical (LO) phonons in $\mathrm{UO}_{2}$ : measurements (blue and red symbols) and simulations (blue and red lines).

Figure 4. SEM micrograph of polycrystalline thin film cross section. MTRM phase profiles under different thermal penetration depth. Multiparameter fitting is implemented to determine thermal conductivity of $\mathrm{Al}$ transducer film and $\mathrm{CeO}_{2}$ film, based on literature values for density and specific heat for $\mathrm{Al}$ and $\mathrm{CeO}_{2}$ and all Si parameters.

Figure 5. Temperature dependent thermal transport in polycrystalline thin films and sintered pellet with corresponding average grain sizes $100 \mathrm{~nm}$ and $5 \mu \mathrm{m}$. Solid lines are model best fits based on BTE time relaxation (Callaway).

Figure 6. Temperature dependence of the thermal conductivity for a single crystal (red), and systems containing one (green) and two (blue) edge dislocations in $\mathrm{UO}_{2}$.

Figure 7. Temperature dependence of the thermal conductivity of $\mathrm{UO}_{2}$ predicted by the Busker potential as calculated with molecular dynamics simulations (open symbols) and with lattice dynamics (closed symbols).

Figure 8 . Thermal conductivity of $\mathrm{UO}_{2}$ at $300 \mathrm{~K}$ predicted by various empirical interatomic potentials (red) compared to the experimental value (blue).

Figure 9. Simulated ballistic transport of phonons in Si single crystal. The hot and cold ends are fixed at $11.88 \mathrm{~K}$ and $3 \mathrm{~K}$, respectively. The temperature profiles along the simulated geometry are plotted at six different times during the simulation. CMSNF's simulation reaches a steady state with a uniform temperature of $10 \mathrm{~K}$ and agrees with the results obtained in Lacroix et al.

Figure 10. Resampled phonon frequencies after scattering computed with the theoretical distributions at $300 \mathrm{~K}$ and $500 \mathrm{~K}$. (a) Current simulation and (b) published results in Lacroix et al.

Figure 11. High-resolution (a) $\mathrm{Ce} 3 \mathrm{~d}$ and (b) $\mathrm{O}$ 1s spectra for ceria film with $40 \%$ oxygen partial pressure.

Figure 12. Cross-section SEM images of $\mathrm{CeO}_{2}$ films deposited on $\mathrm{Si}$ wafer substrates and annealed at temperature of (a) RT (as-deposited), (b) $800^{\circ} \mathrm{C}$, (c) $1000^{\circ} \mathrm{C}$, and (d) $1100^{\circ} \mathrm{C}$. 
Figure 13. Atomic resolution images for $980 \mathrm{MeV}$ Au ion irradiated $\mathrm{CeO}_{2}$ to a fluence of $5 \mathrm{e} 12$ ion $/ \mathrm{cm}^{2}$, (a) an area where Au ion may travel through, and (b) high resolution image of the marked region in (a).

Figure 14. (a) Pair distribution function of as-received and swift heavy ion irradiated $\mathrm{CeO}_{2}$, and (b) calculated lattice parameters for swift heavy ion irradiated $\mathrm{CeO}_{2}$. The results indicate that $\mathrm{CeO}_{2}$ lattice constant increased after swift heavy ion irradiation.

Figure 15. Influence of an extra barrier for defect surface reactions exceeding the migration barrier by $1 \mathrm{eV}$ on the shrinkage of a 2-dimensional void of radius $100 \mathrm{~nm}$ in copper at $1000 \mathrm{~K}$ with initial interstitial supersaturation of $10^{7}$ : (a) interstitial concentration profile after $400 \mathrm{~ns}$ as a function of distance to the void with and without extra barrier for surface reactions; (b) displacement of the void surface as a function of time with and without extra barrier for surface reactions.

Figure 16. Uranium (a) and oxygen (b) interstitial concentration profile near a flat surface of an defected but stoichiometric $\mathrm{UO}_{2}$ crystal with initial concentration of uranium interstitials of $\mathrm{c}_{\mathrm{U}_{1}}=0.0001$ and, vacancies $\mathrm{c}_{\mathrm{Uv}}=0.01$ and according oxygen defects concentrations of $\mathrm{c}_{\mathrm{Oi}}=0.0002$ and $\mathrm{c}_{\mathrm{OV}}=0.01$. The imbalance of defect fluxes causes changes of stoichiometry near the surface [right end of the figures].

Figure 17. Atom probe reconstruction showing $21 \% \mathrm{Ce}$ iso-concentration surface in red. Enclosed in these surfaces are areas where the Ce concentration is higher than $21 \%$. These concentration gradients did not originate from ion damage but from artifacts caused by laser-assisted evaporation.

Figure 18. Atom probe reconstruction of thin film $\mathrm{CeO}_{2}$. Red atoms indicate the location of the $\mathrm{Ce}$ atoms while the $\mathrm{O}$ atoms are colored blue.

\section{TABLES}

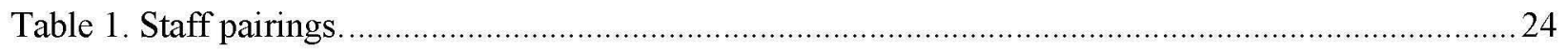

Table 2. FY2011 budget distribution by institution and principal investigator. ....................................2 27

Table 3. Estimated carryover into FY 2011 (in \$K) by institution and principal investigator. .................28 


\section{ACRONYMS}

ACSSEL Advanced Coatings and Surface Engineering Laboratory

AECL Atomic Energy Canada Limited

ANL Argonne National Laboratory

APS Advanced Photon Source

ASCR Advanced Scientific Computing Research

ATR Advanced Test Reactor

BES Basic Energy Science

BTE Boltzmann Transport Equation

CAMS Center for Advanced Modeling and Simulation

CASL Consortium for Advanced Simulation of Light Water Reactors

CMNS Computational Materials Science Network

CMSNF Center for Material Science of Nuclear Fuels

COMB Charge-Optimized Many-body

DOE Department of Energy

EFRC Energy Frontier Research Center

EPMA Electron Probe Micro-analyzer

FSU Florida State University

FWP Field Work Proposal

GSI Gesellschaft für Schwerionenforschung (Germany)

HFIR High-Flux Isotope Reactor

INL Idaho National Laboratory

INS inelastic neutron scattering

IVEM Intermediate Voltage Electro Microscope

KMC kinetic Monte-Carlo

LA longitudinal acoustic

LANL Los Alamos National Laboratory

LD lattice dynamics

LO longitudinal optical

MD molecular dynamic

MIT Massachusetts Institute of Technology

MTRM modulated thermoreflectance microscopy

NE Nuclear Energy

NERI Nuclear Energy Research Institute 


$\begin{array}{ll}\text { NRC } & \text { National Research Council } \\ \text { NSUF } & \text { National Scientific User's Facility } \\ \text { ORNL } & \text { Oakridge National Laboratory } \\ \text { PDF } & \text { pair distribution functions } \\ \text { P-UBMS } & \text { pulsed, unbalanced magnetron sputtering } \\ \text { RTA } & \text { rapid thermal annealing } \\ \text { SciDAC } & \text { Scientific Discovery through Advanced Computing Program } \\ \text { SEM } & \text { scanning electron microscopy } \\ \text { SRM } & \text { series resistance model } \\ \text { T } & \text { temperature } \\ \text { TA } & \text { transverse acoustic } \\ \text { TEM } & \text { transmission electron microscope } \\ \text { TMS } & \text { The Minerals, Metals and Materials Society } \\ \text { TOPS } & \text { Terascale Optimal PDE Simulations } \\ \text { UF } & \text { University of Florida } \\ \text { UWM } & \text { University of Wisconsin-Madison } \\ \text { XPS } & \text { x-ray spectroscopy } \\ \text { XRD } & \text { x-ray diffraction }\end{array}$




\section{Energy Frontier Research Center, Center for Materials Science of Nuclear Fuels}

\section{TECHNICAL ACCOMPLISHMENTS}

The science driver of the Center for Material Science of Nuclear Fuels' (CMSNF) investigation is to understand how complex defect and microstructures affect phonon-mediated thermal transport in $\mathrm{UO}_{2}$, and achieve this understanding for the particular case of irradiation-induced defects and microstructures. The center's research includes modeling and measurement of thermal transport in oxide fuels with different levels of impurities, lattice disorder, and irradiation-induced microstructure, as well as theoretical and experimental investigation of the evolution of disorder, stoichiometry, and microstructure in nuclear fuel under irradiation. With the premise that thermal transport in irradiated $\mathrm{UO}_{2}$ is a phononmediated energy transport process in a crystalline material with defects and microstructure, a step-by-step approach will be utilized to understand the effects of types of defects and microstructures on the collective phonon dynamics in irradiated $\mathrm{UO}_{2}$.

\subsection{Thermal Transport}

CMSNF's efforts under the thermal transport thrust involved both measurement of diffusive phonon transport (an approach that integrates over the entire phonon spectrum) and spectroscopic measurements of phonon attenuation/lifetime and phonon dispersion. The center's distinct experimental efforts dovetail with the thust's modeling effort involving atomistic simulation of phonon transport and prediction of lattice thermal conductivity using the Boltzmann transport framework.

\subsubsection{Spectroscopy of Phonon Dynamics}

\subsubsection{Inelastic Neutron Scattering Measurements of Phonon Dispersion and Line widths in $\mathrm{UO}_{2}$}

To obtain a fundamental understanding of thermal conductivity in $\mathrm{UO}_{2}$ as a prototype nuclear fuel, high-resolution $(<1 \mathrm{meV})$ inelastic neutron scattering measurements of phonon line widths at room temperature $(295 \mathrm{~K})$ and at $1200 \mathrm{~K}$ have been initiated. Measurements of phonon energies and spectroscopic line widths were performed for transverse and longitudinal acoustical modes and selected optical modes along the [001] and [111] directions in single crystal $\mathrm{UO}_{2}$ using the $\mathrm{HB} 3$ triple-axis spectrometer at the High-Flux Isotope Reactor (HFIR) at Oak Ridge National Laboratory (ORNL). As illustrated in Figure 1, the magnitude of phonon energy shifts and phonon line width increases due to anharmonic effects were measured as a function of wave vector $q$. These measurements represent the first high-resolution measurements of phonon line widths in $\mathrm{UO}_{2}$, and provide direct and fundamental information on thermal transport by individual phonons and phonon modes in $\mathrm{UO}_{2}$.

Accordingly, by combining measured phonon group velocities (determined from energy dispersion gradients) with measured phonon lifetimes (inverse phonon line widths) it is now

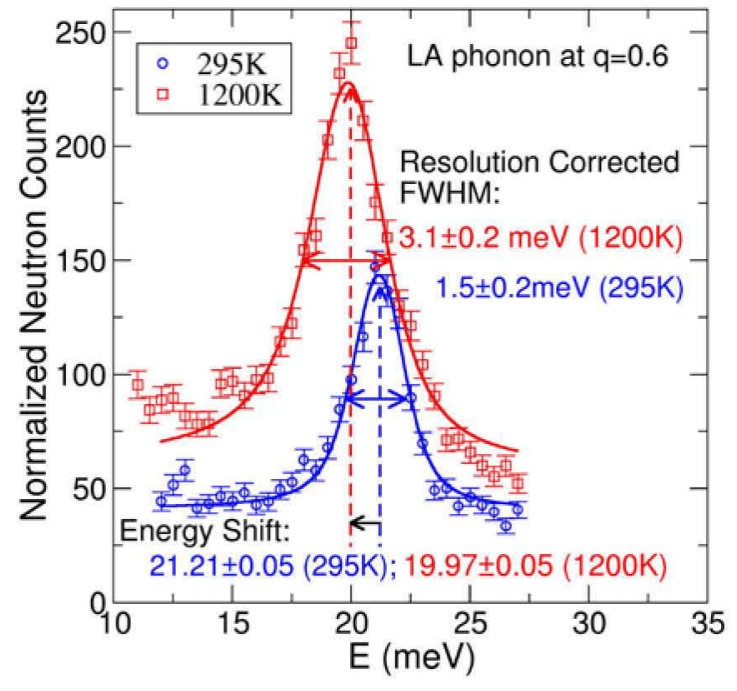

Figure 1. Longitudinal acoustic phonons in $\mathrm{UO}_{2}$ measured for temperatures of $295 \mathrm{~K}$ and $1200 \mathrm{~K}$; the solid lines are fits to the data. 
possible to compare thermal transport by transverse versus longitudinal polarization phonons and optical versus acoustical phonon branches and as a function temperature. Moreover, these data provide direct and absolute basis for comparison with theory and simulations within the CMSNF.

\subsubsection{Contribution of $\mathrm{UO}_{2}$ Phonon Branches to Thermal Conductivity}

Key to the fundamental understanding of thermal conductivity is how heat conduction is partitioned between phonon modes. An analysis of inelastic neutron scattering measurements of phonon line widths and phonon energy dispersion gradients shows that transverse acoustic (TA) and longitudinal optical (LO) phonons (see Figure 2) in $\mathrm{UO}_{2}$ transport heat in amounts similar to that of the longitudinal acoustic (LA) phonons. This surprising result is contrary to conventional expectations based on the fact that longitudinal acoustic phonon velocities are twice that of the transverse acoustical modes as indicated by the stronger dispersion in Figure 2. However, the lifetimes for LA mode phonons were found to be less than half that for TA phonons in $\mathrm{UO}_{2}$ for large wave vectors.

Moreover, the implication that the LO modes have high thermal transport is in stark contrast to recently reported first principles dynamical mean field theory simulations, which concluded using phonon branch-specific Grüneisen analyses that LA phonons are responsible for more than $90 \%$ of thermal conductivity of $\mathrm{UO}_{2}$ at $1000 \mathrm{~K}$ (Yin and Savrasov 2008).

The ability to extract fundamental information on individual phonon mode contributions to thermal transport directly from inelastic neutron measurements provides a previously unavailable, direct, and absolute benchmark for theory and simulations within the CMSNF.

\subsubsection{Lifetimes of $\mathrm{LO}$ phonons in $\mathrm{UO}_{2}$}

To test the understanding of the fundamental aspects of thermal transport in $\mathrm{UO}_{2}$, direct comparisons have been made between inelastic neutron scattering (INS) measurements and lattice dynamics (LD) simulations of the spectroscopic line widths for the high-velocity longitudinal optical branch phonons as a function of temperature. As shown in Figure 3, strong, non-monotonic variations are present in both the INS measurements and the LD simulations, especially at high temperature. CMSNF researchers note further that the LD simulations are in remarkably good agreement with the measurements for all wave vectors $(q)$ at $295 \mathrm{~K}$. Although the predicted phonon lifetimes (i.e., inverse line widths) differ by as much as a factor of two from the measurements in the mid- $q$ region at $1200 \mathrm{~K}$, strong $q$-dependent variations are found at high temperature for both the LD simulations and the INS measurements.

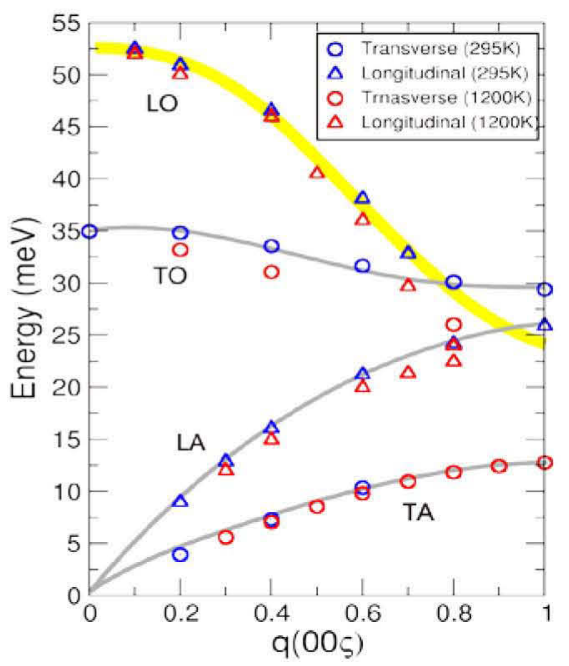

Figure 2. Measured dispersion curves for $\mathrm{UO}_{2}$ at $295 \mathrm{~K}$ and $1200 \mathrm{~K}$. Circles (triangles) represent transverse (longitudinal) phonons. Blue (red) symbols denote measurements at $295 \mathrm{~K}(1200 \mathrm{~K})$. The lines are guides to the eye and the highlighted line denotes the high-velocity longitudinal optical phonon branch.

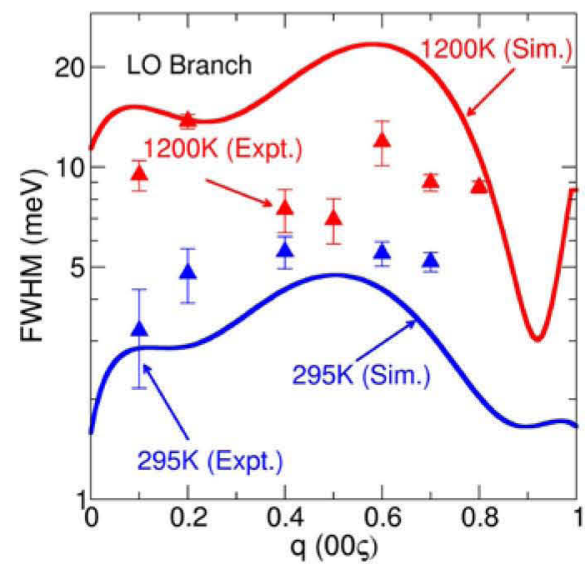

Figure 3. Line widths for longitudinal optical (LO) phonons in $\mathrm{UO}_{2}$ : measurements (blue and red symbols) and simulations (blue and red lines). 
Since anharmonicity increases strongly with temperature and anharmonic phonon-phonon scattering processes depend strongly on the initial phonon momentum, the enhanced wave vector dependence in phonon lifetimes at high temperature can be expected qualitatively. Moreover, the fact that the full Brillouin zone LD calculations, which are based on perturbation theory and take these effects into account quantitatively, predict strong wave vector dependence at high temperature supports this analysis. Although improved potentials are apparently needed for LD calculations, these new measurement and simulation results rule out the common assumption in earlier phonon transport theories that phonon lifetime is a function of temperature and phonon energy only.

\subsubsection{Thermal Conductivity Measurements}

\subsubsection{Spatially Resolved Thermal Transport in Surrogate Nuclear Fuel}

The influence of grain boundaries on thermal transport in surrogate nuclear fuel samples is being analyzed. Nuclear fuel has a very complex and spatially varying microstructure. The rim region of the fuel pellet can be composed of submicron grains, and it is thought that the thermal resistance posed by grain boundaries in this region of the fuel can play a significant role in limiting thermal transport.

Modulated thermoreflectance microscopy (MTRM) was used to measure thermal transport in polycrystalline ceria thin films grown on Si substrates. MTRM is a nondestructive laser-based thermal wave imaging technique that provides submicron resolution and is well suited for measurement of heterogeneous samples. Examples of the types of samples suited for measurement by MTRM are thin film geometries, ion-irradiated samples where only a thin layer near the surface is influenced by displacement damage and nuclear fuel where the microstructure and thermal transport properties are strong functions of the radial distance from the fuel center.

Polycrystalline ceria thin films (used as a surrogate for $\mathrm{UO}_{2}$ ) were deposited onto $\mathrm{Si}$ substrates using pulsed, unbalanced magnetron sputtering (P-UBMS) from a cerium target in a reactive $\mathrm{Ar}-\mathrm{O}_{2}$ environment to produce a range of $\mathrm{Ce}-\mathrm{O}$ stoichiometries that varied from mixed $\mathrm{CeO}_{2}-\mathrm{Ce}_{2} \mathrm{O}_{3}$ to stoichiometric $\mathrm{CeO}_{2}$. The thickness of the films varied from $500 \mathrm{~nm}$ to $10 \mu \mathrm{m}$ and the columnar grain widths varied from $5 \mathrm{~nm}$ to almost $1 \mu \mathrm{m}$. Scanning electron microscopy (SEM) images combined with $\mathrm{x}$-ray diffraction analysis were used to reveal the grain structure and $\mathrm{Ce}-\mathrm{O}$ phases presented in the $\mathrm{Ce}-\mathrm{O}$ films. Figure $4 \mathrm{a}$ is a SEM image of a $\mathrm{CeO}_{2}$ film with columnar grains having an average diameter of $100 \mathrm{~nm}$. An analysis routine has been developed that measures the thermal wave profile under a series of

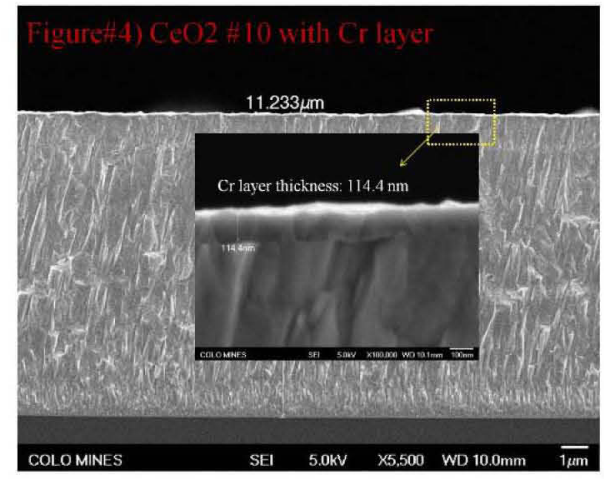

(a)

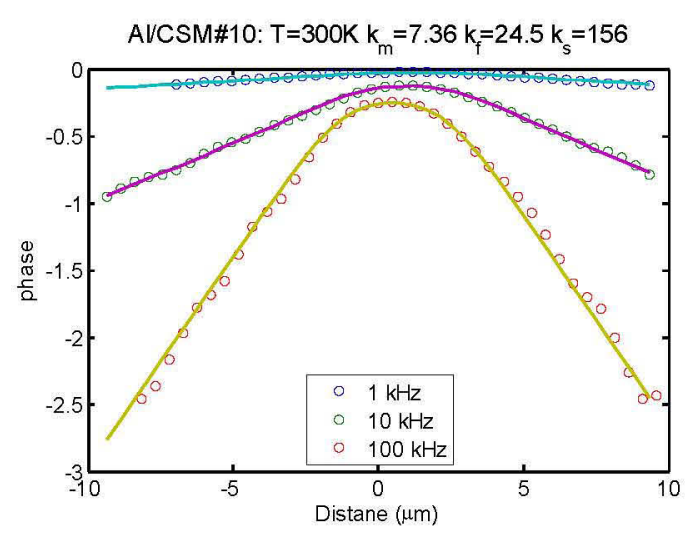

(b)

Figure 4. SEM micrograph of polycrystalline thin film cross section. MTRM phase profiles under different thermal penetration depth. Multiparameter fitting is implemented to determine thermal conductivity of Al transducer film and $\mathrm{CeO}_{2}$ film, based on literature values for density and specific heat for $\mathrm{Al}$ and $\mathrm{CeO}_{2}$ and all Si parameters. 
pump modulation frequencies and performs multiparameter least squares fitting to a heat conduction model to extract thermal conductivities of the ceria film and Al transducer film (Figure $4 \mathrm{~b}$ ). ${ }^{2}$ The measured thermal conductivity of the ceria thin film analyzed is $7.4 \mathrm{~W} / \mathrm{mK}$ at T $=300 \mathrm{~K}$.

To understand the influence of grain boundaries on thermal transport, phonon mean-free path was varied relative to a fixed grain size by performing experiments at cryogenic temperatures. Using the fitting routine, the temperature $(\mathrm{T})$ dependent conductivity of the ceria thin film was determined (see Figure 5, red circles), where literature values for T-dependent conductivity of $\mathrm{Si}$ and specific heat values for $\mathrm{Al}, \mathrm{CeO}_{2}$, and $\mathrm{Si}$ were used. The density of all elements was assumed to be T-independent. Thermal conductivity of the sample increases with increasing temperature. Comparing this with a similar work on polycrystalline, ${ }^{3}$ the thermal transport in the measured T-range is determined to be limited by scattering at grain boundaries.

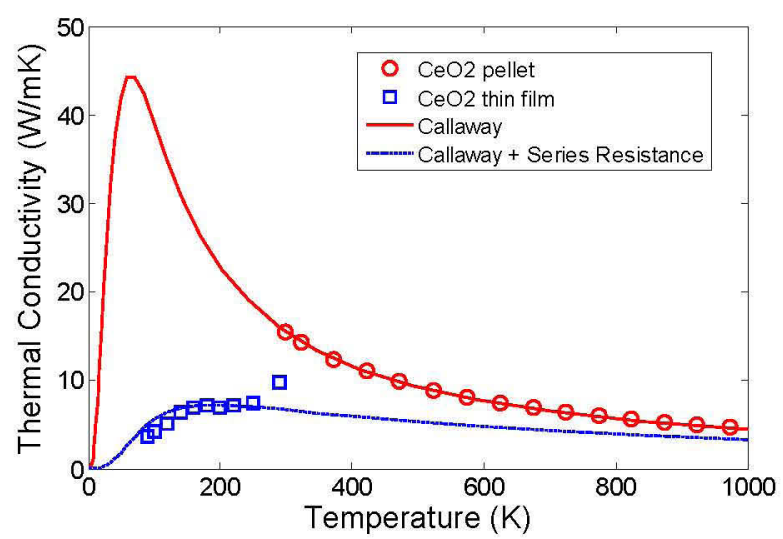

Figure 5. Temperature dependent thermal transport in polycrystalline thin films and sintered pellet with corresponding average grain sizes $100 \mathrm{~nm}$ and $5 \mu \mathrm{m}$. Solid lines are model best fits based on BTE time relaxation. ${ }^{3}$

The thermal conductivity of a polycrystalline thin film was compared to that of a sintered pellet that has a larger grain size, on the order of $5 \mu \mathrm{m}$. Conductivity of the $\mathrm{CeO}_{2}$ pellet was determined from diffusivity measurements using a laser flash apparatus (see Figure 5, blue squares). At $\mathrm{T}=300 \mathrm{~K}, \mathrm{k}=$ $15.6 \mathrm{~W} / \mathrm{mK}$, the conductivity decreases with increasing $\mathrm{T}$ and has a 1/T-type dependence, typical of transport governed by intrinsic phonon-phonon scattering.

Finally, the experimental results are compared with the predictions of Boltzmann transport formalism. The strength of the phonon-phonon (ph-ph) scattering within the relaxation time approximation was extracted from the fit of high-T conductivity data from the sintered sample. ${ }^{3}$ The same ph-ph scattering together with series resistance model (SRM) to account for grain boundary scattering was used to fit the experimental data observed in polycrystalline thin film. In SRM, the Kapitza boundary is calculated at each $\mathrm{T}$ using a frequency-dependent scattering probability and grain size as fitting parameters. A good fit was obtained between model and experiment, but there is a disagreement between fitted grain size and experimentally grain size determined from the SEM image.

In conclusion, the measured thermal transport in polycrystalline thin films and sintered pellets was measured for $\mathrm{CeO}_{2}$. Thermal transport is governed by grain boundary scattering and intrinsic phononphonon scattering in the latter. Further modeling efforts are needed to understand thermal transport in polycrystalline thin films.

\subsubsection{Atomistic Simulation of Thermal Transport Properties}

The objective of this effort is the reliable and efficient prediction of the thermal transport properties of $\mathrm{UO}_{2}$, including the effects of microstructure. Significant progress has been made in three complementary tasks.

\section{Task 1: Effect of Dislocations on Thermal-transport Properties}

The microstructure of the $\mathrm{UO}_{2}$ fuel pellet evolves continuously during operation. Therefore, it is important to understand the effects of different microstructural elements on the thermal-transport properties. The effects of edge and screw dislocations on the thermal-transport properties of $\mathrm{UO}_{2}$ have 
been characterized. Figure 6 shows that the effect of a dislocation on the thermal conductivity is proportional to the concentration and is essentially temperature independent. Both of these conclusions are consistent with qualitative understanding from simple analytic analyses. Current work is focused on distinguishing between the effects of edge and screw dislocations and on putting these in the context of the microstructural evolution during burnup.

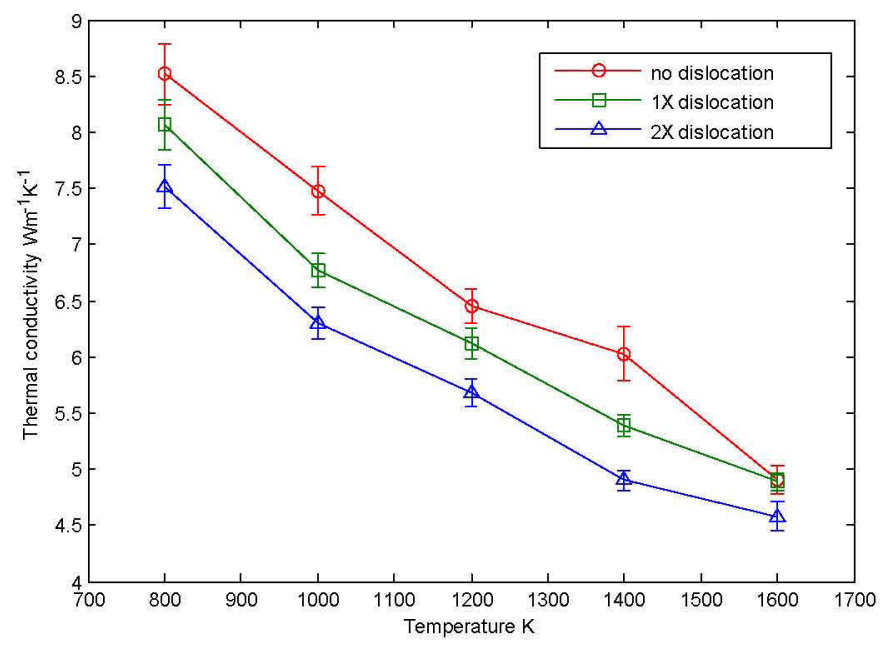

Figure 6. Temperature dependence of the thermal conductivity for a single crystal (red), and systems containing one (green) and two (blue) edge dislocations in $\mathrm{UO}_{2}$.

\section{Task 2: Calculation of thermal conductivity from three-phonon processes}

An analytic lattice-dynamics based method for the calculation of the thermal conductivity has been developed. The thermal conductivity of bulk systems is dominated by three-phonon processes: one phonon splitting into two, or two phonons combining into one. The effects of these processes on the thermal transport properties were calculated at the level of perturbation theory in lattice dynamics, using an atomiclevel Boltzmann transport equation approach. By comparing the results of this analytic approach with the results of moleculardynamics simulations, it was established that the analytic approach is reliable, and that the three-phonon processes are dominant (see Figure 7). This method was then used to calculate the thermal transport properties of $\mathrm{UO}_{2}$ as described by a number of literature

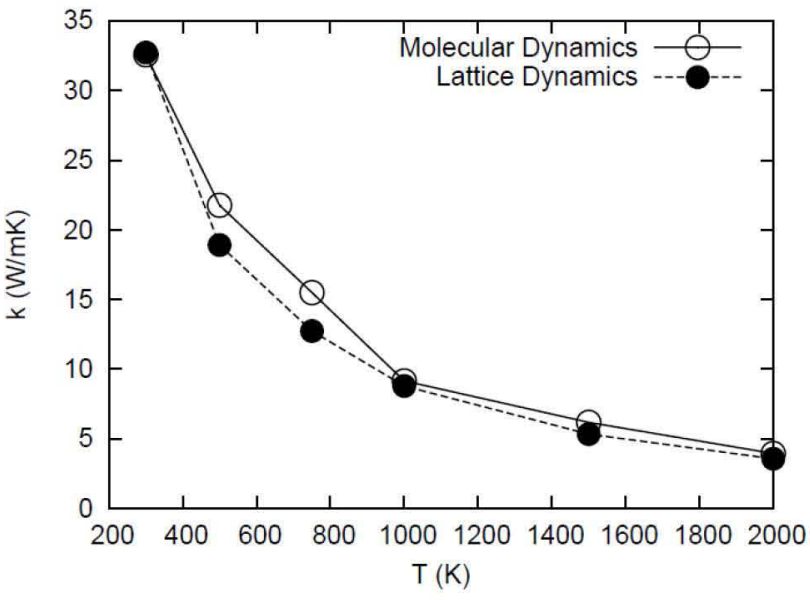

Figure 7. Temperature dependence of the thermal conductivity of $\mathrm{UO}_{2}$ predicted by the Busker potential as calculated with molecular dynamics simulations (open symbols) and with lattice dynamics (closed symbols). empirical interatomic potentials. The anharmonic effects that control the thermal conductivity have not, in the past, been part of the training data set for developing the interatomic potentials. As a result, and as shown in Figure 8 on the following page, some potentials yield thermal conductivities that agree well with experimental values, while others give significant overestimates, indicating that the anharmonic effects are significantly underestimated. 


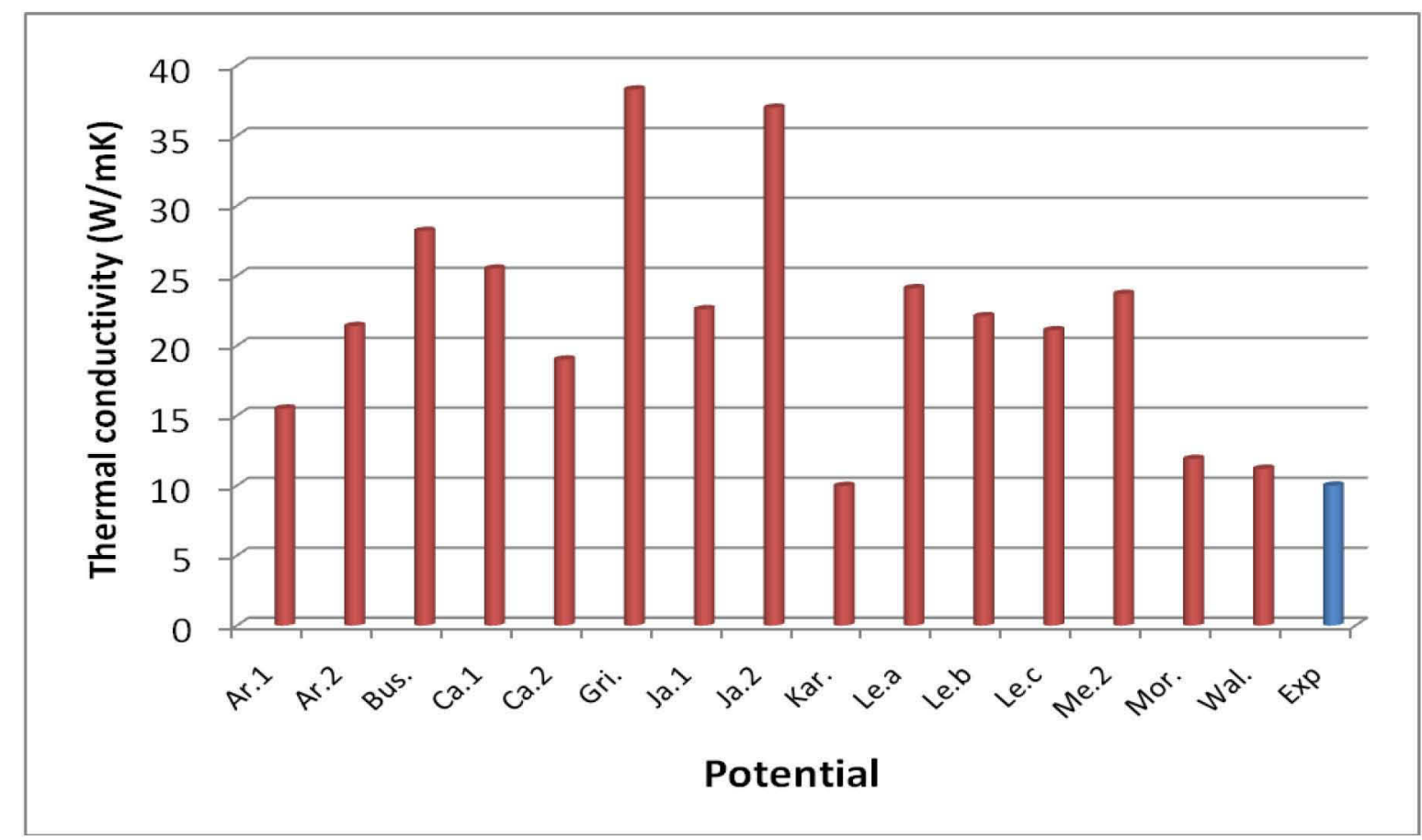

Figure 8. Thermal conductivity of $\mathrm{UO}_{2}$ at $300 \mathrm{~K}$ predicted by various empirical interatomic potentials (red) compared to the experimental value (blue).

\section{Task 3: High-fidelity interatomic potentials for $\mathrm{U}$ and $\mathrm{UO}_{2}$}

There are a large number of empirical potentials for $\mathrm{UO}_{2}$, none of which is able to reproduce all of the key physical properties (energetics, elasticity, defect properties, thermal expansion, thermal transport) with high fidelity. The origin of this lack of materials fidelity seems to lie in the limited amount of physics encoded in the potential forms used. The formalism of Charge-Optimized many-body (COMB) potentials, developed at the University of Florida (UF), is being used to develop new, high-fidelity interatomic potentials for $\mathrm{UO}_{2}$ and related systems. As part of this process, an interatomic description for $\mathrm{U}$ metal is also being developed, for which there are no potentials in the literature. First results are highly encouraging; further optimization is ongoing.

\subsubsection{Monte Carlo Solution of the Boltzmann Transport Equation}

A Monte Carlo technique has been implemented to solve Boltzmann Transport Equation (BTE) for phonons. The technique is based on the relaxation time approximation, where the full BTE is simplified by assigning time scales to each scattering mechanism associated with phonon interactions. Currently only phonon-phonon interactions are modeled, although boundary and impurity scatterings are simple extensions.

At this early phase of implementation of this BTE solution technique, the focus is on reproducing published results for $\mathrm{Si}$, with a planned extension to consider the full range of phonon processes in $\mathrm{UO}_{2}$. The ballistic regime of phonon transport in silicon has been successfully reproduced. Figure 9 shows the temperature profile along a simulated geometry at several different time steps. The left of the simulated domain is held fixed at $11.88 \mathrm{~K}$ while the right end is fixed at $3 \mathrm{~K}$. At such low temperatures, scattering events are negligible. The simulation reaches steady state with a uniform temperature profile at the theoretical temperature of $10 \mathrm{~K}$. The $500 \mathrm{ps}$ and the $1 \mathrm{~ns}$ curves can be seen as containing two components. The faster and slower components travel according to the longitudinal and transverse group velocities, respectively. This has been compared to the same result obtained by Lacroix et al. ${ }^{4}$ 


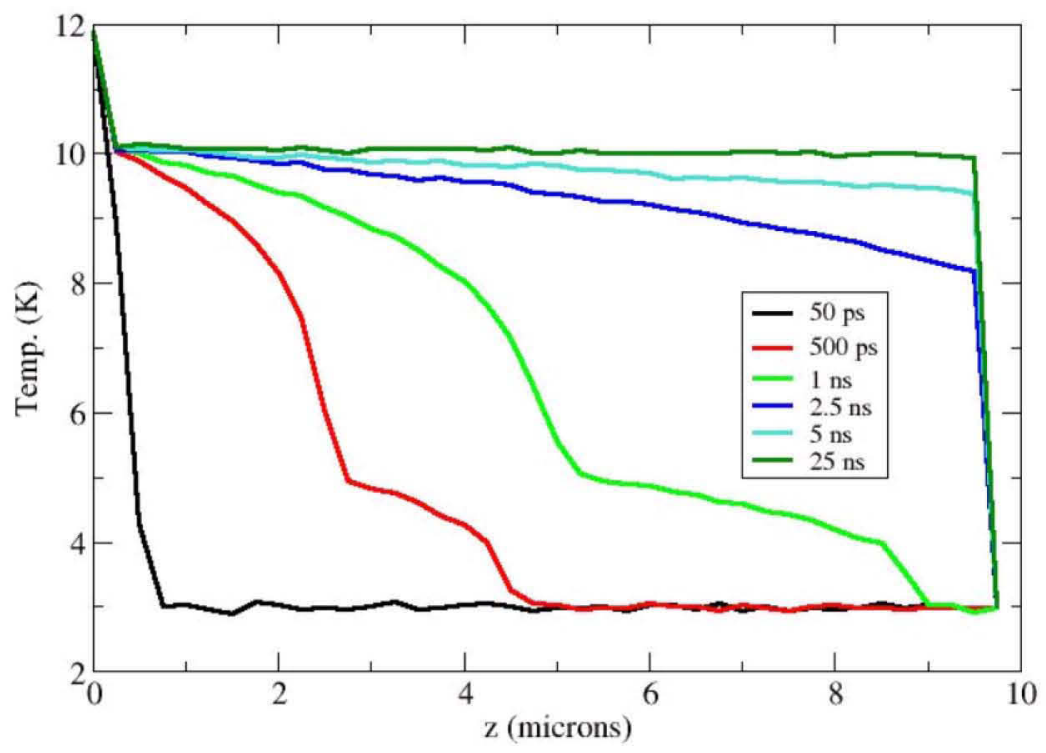

Figure 9. Simulated ballistic transport of phonons in Si single crystal. The hot and cold ends are fixed at $11.88 \mathrm{~K}$ and $3 \mathrm{~K}$, respectively. The temperature profiles along the simulated geometry are plotted at six different times during the simulation. CMSNF's simulation reaches a steady state with a uniform temperature of $10 \mathrm{~K}$ and agrees with the results obtained in Lacroix et al.

As the simulation is run at increasingly higher temperatures, phonon-phonon scattering rates increase accordingly. When a phonon is scattered, its energy/frequency and direction are re-sampled. Unlike true phonon scattering processes, as phonons scatter in the Monte Carlo simulation, phonon numbers are conserved while phonon energies are not. To conserve energy in the system, a method has been adopted from Lacroix et al. ${ }^{4,5}$ for re-sampling phonon energies after a scattering event. This algorithm enforces Kirchoff's law for phonons since phonons of a given frequency are re-sampled into the population at the same rate that they are removed. Currently, the researchers are seeing discrepancies in how phonon energies are redistributed after scattering when compared to results obtained by Lacroix et al. ${ }^{5}$ Figure 10 (a) and 2(b) compare the re-sampled phonon frequencies to the theoretical distribution at $300 \mathrm{~K}$ and $500 \mathrm{~K}$. Note in Figure 10(a) that the frequencies seem to get slightly shifted toward the transverse cut-off frequency. This tends to cause the simulations to slightly lose energy as it steps in time. The resolution of this issue is being investigated before moving on to $\mathrm{UO}_{2}$ simulations. After resolving the energy conservation in silicon simulations, the only changes needed in the current model are dispersion curves and the expressions used to calculate the phonon-phonon scattering rates. $\mathrm{UO}_{2}$ dispersion curves are readily available in the literature and have been measured recently as part of this EFRC project. Relaxation time expressions similar to those developed by Han and Klemens ${ }^{6}$ are planned for the implementation of the $\mathrm{UO}_{2}$ Monte Carlo. 


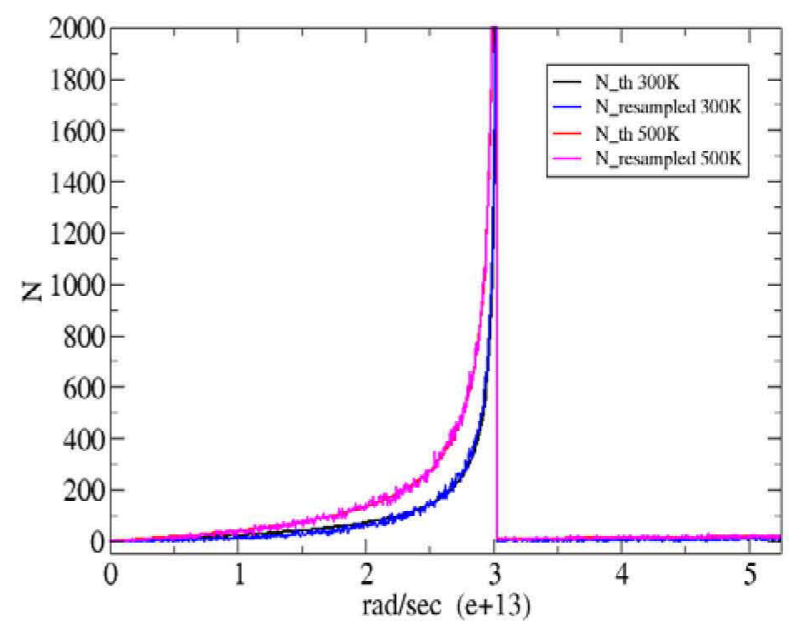

(a)

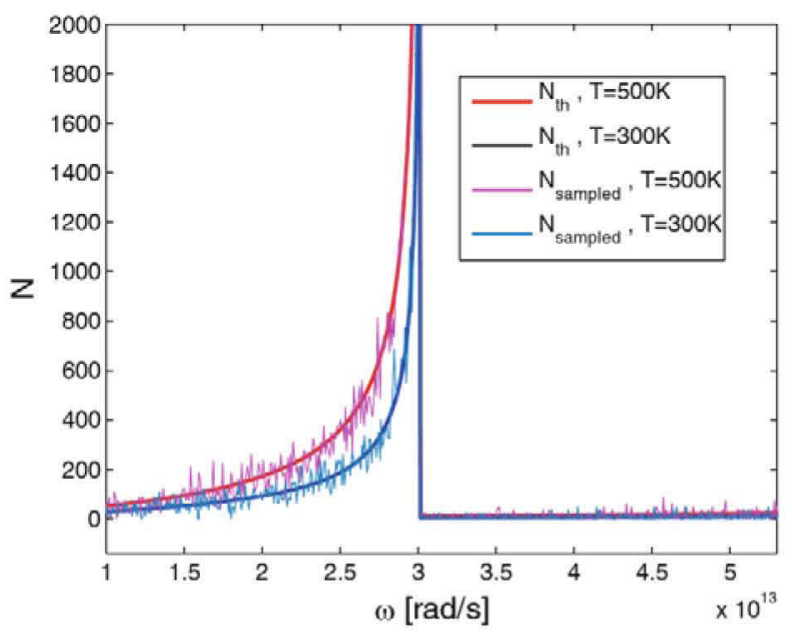

(b)

Figure 10. Resampled phonon frequencies after scattering computed with the theoretical distributions at $300 \mathrm{~K}$ and $500 \mathrm{~K}$; (a) Current simulation and (b) published results in Lacroix et al.

\subsection{Microstructure under Radiation Coupled to Thermal Transport}

\subsubsection{Preparation of Thin-Film Samples for Irradiation and Thermal Transport Measurements}

Ceria films, used as a $\mathrm{UO}_{2}$ surrogate, were deposited on silicon wafers in an argon-oxygen atmosphere using P-UBMS from a metallic Ce target. Ceria films were annealed using rapid thermal annealing (RTA) in the range from room temperature to $1100^{\circ} \mathrm{C}$. Compositional analyses of the films to determine the atomic content of $\mathrm{Ce}, \mathrm{O}$, and $\mathrm{Si}$ (impurity) were carried out by electron probe microanalyzer (EPMA). All of the ceria films have slightly higher cerium atomic percent contents (i.e., lower oxygen at.\% relatively) than that of the $\mathrm{Ce}$ atomic percent of 33.33 at.\% required in stoichiometric $\mathrm{CeO}_{2}$ films. The x-ray diffraction (XRD) patterns of all ceria films exhibited a crystalline cubic $\mathrm{CeO}_{2}$ phase with preferred orientations of the (111) and (222) with small weak peaks of the (220) and (311) crystallographic planes. Small XRD peaks corresponding to crystalline hexagonal $\mathrm{Ce}_{2} \mathrm{O}_{3}$ phase for (100) plane were also observed. X-ray photoelectron spectroscopy (XPS) was performed to confirm the chemical state of $\mathrm{Ce}(\mathrm{Ce} 4+, \mathrm{Ce} 3+$, etc.) and $\mathrm{O}$ of the ceria samples, as shown in Figure 11.

Based on the XRD, EPMA and XPS results, it is concluded that as-deposited ceria films have a composite microstructure consisting of a large amount of $\mathrm{CeO}_{2}$ columnar grains and a very small amount of $\mathrm{Ce}_{2} \mathrm{O}_{3}$ grains. Figure 12 shows the cross-section SEM images of $\mathrm{CeOx}$ films deposited on $\mathrm{Si}$ wafer substrates and subjected to RTA at various temperatures in a slightly enriched oxygen low-pressure environment. All of the films exhibited a dense columnar structure with grain size in the range of $169 \mathrm{~nm}$ to $500 \mathrm{~nm}$. The crystallinity of the annealed samples was characterized by XRD. The XRD results indicated that the as-deposited ceria films have a cubic $\mathrm{CeO}_{2}$ phase with a (111) preferred orientation. However, post-annealed ceria films showed the presence of a hexagonal $\mathrm{Ce}_{2} \mathrm{O}_{3}$ phase with a $(002)$ preferred orientation after RTA at $1000^{\circ} \mathrm{C}$ for 5 minutes. 

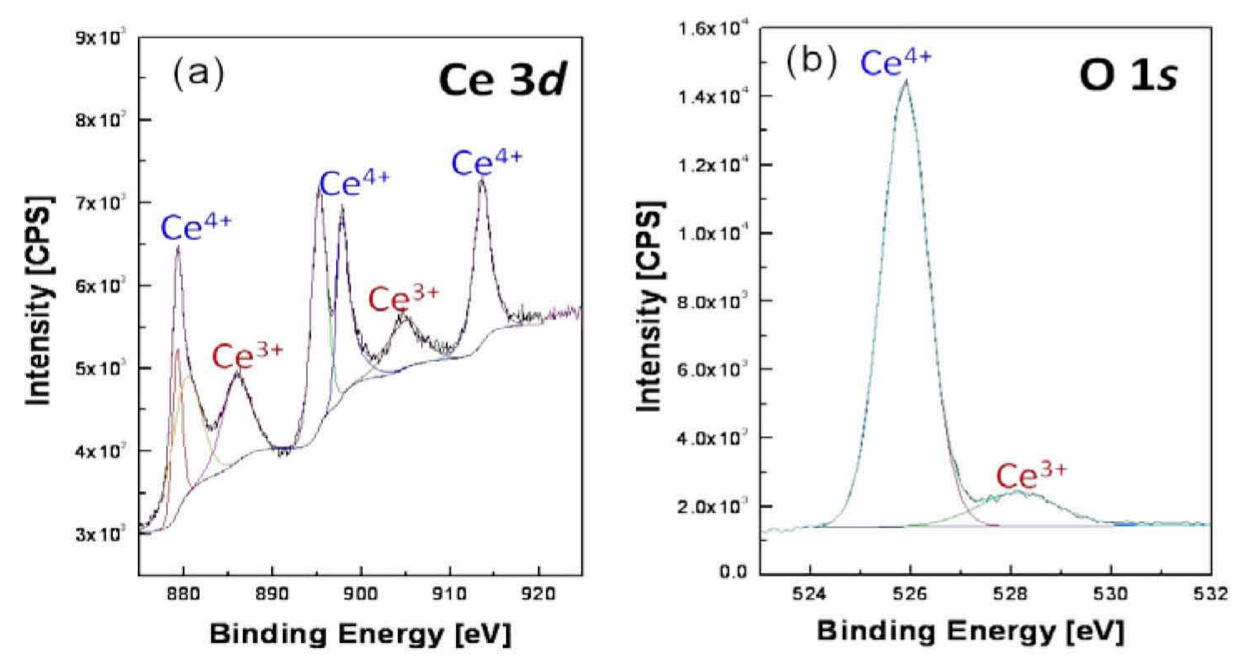

Figure 11. High-resolution (a) $\mathrm{Ce} 3 \mathrm{~d}$ and (b) $\mathrm{O}$ 1s spectra for ceria film with $40 \%$ oxygen partial pressure.

This phase transformation from cubic $\mathrm{CeO}_{2}(111)$ to hexagonal $\mathrm{Ce}_{2} \mathrm{O}_{3}(002)$ is likely due to the $\mathrm{Ce}^{4+}$ to $\mathrm{C}^{\mathrm{e} 3+}$ cation transformation by the formation of oxygen vacancies. In addition, the intensity of the hexagonal $\mathrm{Ce}_{2} \mathrm{O}_{3}$ phase increased with an increase in the post-deposition annealing temperature.
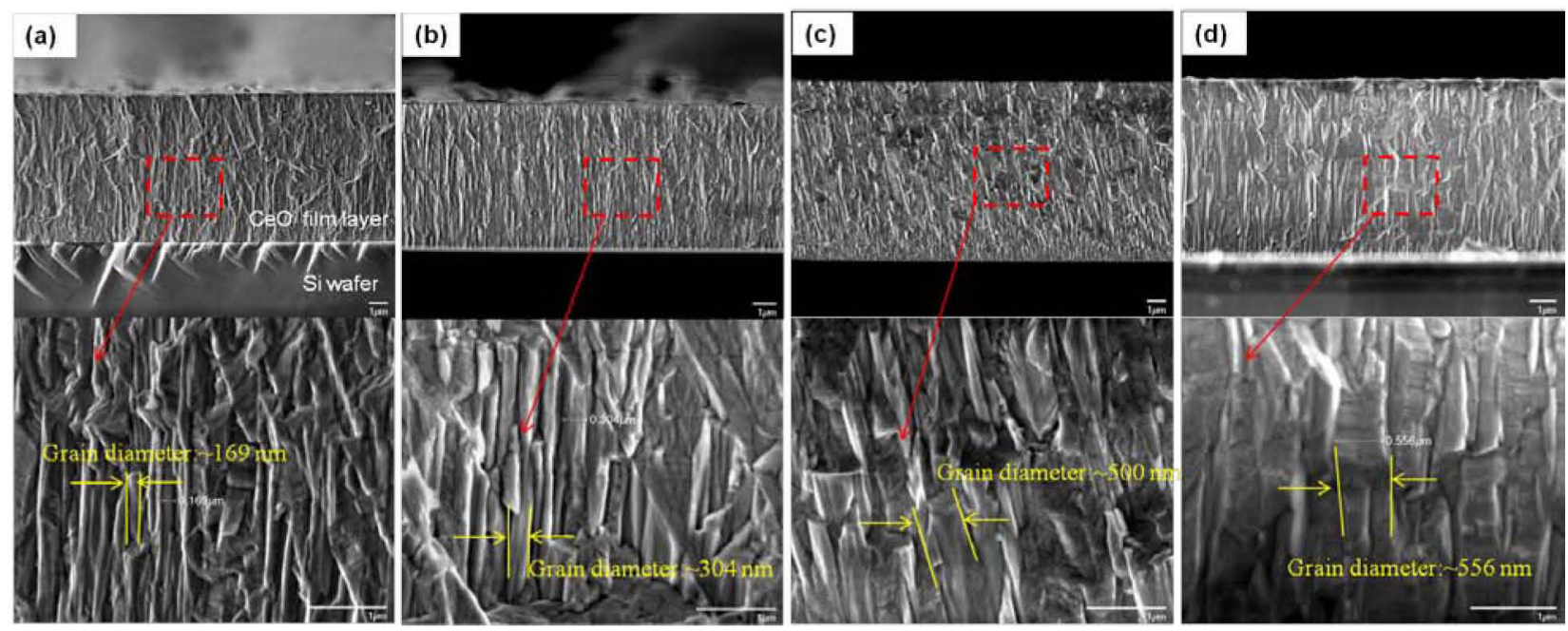

Figure 12. Cross-section SEM images of $\mathrm{CeO}_{2}$ films deposited on $\mathrm{Si}$ wafer substrates and annealed at temperature of (a) RT (as-deposited), (b) $800^{\circ} \mathrm{C}$, (c) $1000^{\circ} \mathrm{C}$, and (d) $1100^{\circ} \mathrm{C}$.

\subsubsection{Irradiation Experiments}

To simulate fission product damage and to separate the effect of electron excitation from ballistic collisions, swift heavy ion irradiation was performed at room temperature on $\mathrm{CeO}_{2}$, chosen as a surrogate material for $\mathrm{UO}_{2}$. $\mathrm{CeO}_{2}$ was found to be resistant to swift heavy ion damage, and no amorphous tracks were formed after both $370 \mathrm{MeV}$ and $980 \mathrm{MeV} \mathrm{Au}$ ion irradiations to a maximum fluence of $5 \mathrm{e} 12$ ions $/ \mathrm{cm} 2$ at room temperature. Figure 13(a) shows a top-view (looking at sample material in a direction that the beam penetrated) transmission electron microscope (TEM) image of the specimen irradiated with $980 \mathrm{MeV}$ Au ions to a fluence of $5 \mathrm{e} 12 \mathrm{ions} / \mathrm{cm} 2$. Based on the fluence, it is expected about five ions on average traveled through the imaged area. Figure 13(b) shows the atomic resolution TEM image of the marked region in Figure 13(a) where one or more Au ions may travel. As the image shows $\mathrm{CeO}_{2}$ retains good crystallinity and there is no sign of amorphization occurring from the irradiation. 


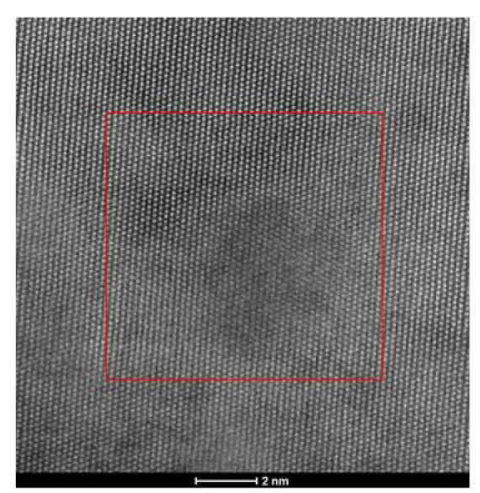

(a)

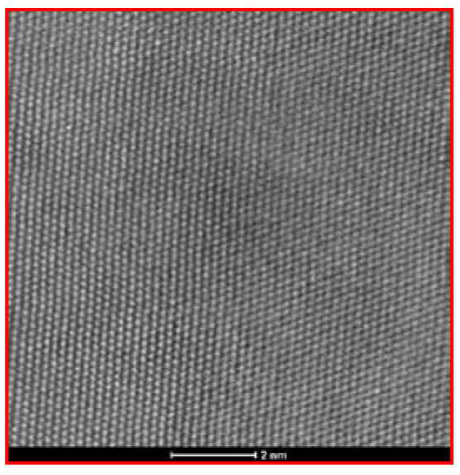

(b)

Figure 13. Atomic resolution images for $980 \mathrm{MeV} \mathrm{Au}$ ion irradiated $\mathrm{CeO}_{2}$ to a fluence of $5 \mathrm{e} 12 \mathrm{ion} / \mathrm{cm}^{2}$, (a) an area where Au ion may travel through, and (b) high resolution image of the marked region in (a).

To determine if there is any local disordering in irradiated $\mathrm{CeO}_{2}$, high energy x-ray scattering was performed for both non-irradiated and irradiated $\mathrm{CeO}_{2}$ in the Advanced Photon Source (APS) at Argonne National Laboratory (ANL). Pair distribution functions (PDF) were obtained after Fourier transformation and are plotted in Figure 1414(a). Structure changes were mainly observed in a short range of less than $1 \mathrm{~nm}$, while the long-range order was well maintained for $\mathrm{CeO}_{2}$ after irradiation. It is also observed that the oxygen sublattices experience more disordering compared to cerium sublattices. Figure 14(b) shows the lattice constants calculated based on the scattering data, and the results indicate that the $\mathrm{CeO}_{2}$ crystal lattice expands after irradiation.

The lattice expansion is most likely due to radiation-induced oxygen defects and the formation of $\mathrm{Ce}^{3+}$ ions. The $\mathrm{Ce}^{3+}$ ion is a bigger ion compared to the $\mathrm{Ce}^{4+}$ ion, which causes the lattice expansion. Determination of the defect structures is ongoing through the refinements of the experimental PDFs. The candidate defect structures will be obtained from literature data and molecular dynamic (MD) simulations performed at Idaho National Laboratory (INL). INL will also perform laser-based thermal transport measurements on the irradiated $\mathrm{CeO}_{2}$ samples and the changes in thermal conductivity will be correlated to the irradiated microstructure. Room temperature $\mathrm{Xe}$ implantation and post annealing in $\mathrm{CeO}_{2}$ thin films with different grain sizes has also been completed. Post annealing examination will focus on the effect of grain boundaries on Xe bubble clustering and distribution. The microstructural analysis will be performed using both transmission electron microscopy and atom probe.

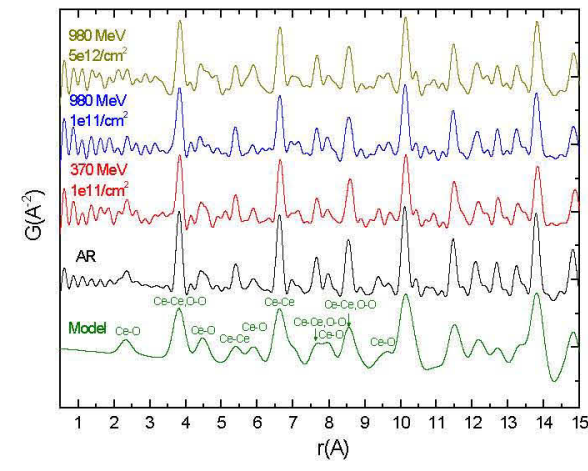

(a)

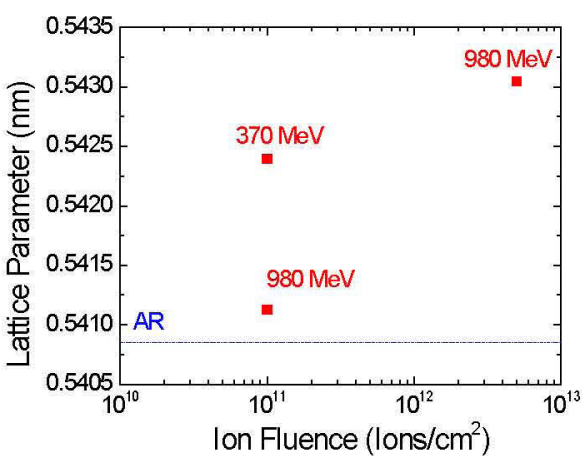

(b)

Figure 14. (a) Pair distribution function of as-received and swift heavy ion irradiated $\mathrm{CeO}_{2}$, and (b) calculated lattice parameters for swift heavy ion irradiated $\mathrm{CeO}_{2}$. The results indicate that $\mathrm{CeO}_{2}$ lattice constant increased after swift heavy ion irradiation. 


\subsubsection{Defect and Microstructure Modeling}

Investigating thermal transport in irradiated $\mathrm{UO}_{2}$ requires a detailed understanding of the defect and microstructure evolution under irradiation. For this purpose, CMSNF researchers are developing a phase field theory for defects and microstructure dynamics and stoichiometry changes in irradiated $\mathrm{UO}_{2}$. Effort focused on the development of the phase field approach for void formation under irradiation, along with the theoretical sharp-interface underpinning for this approach. The work focused on the interactions of vacancies and interstitials at the void surface. Consideration of the appropriate kinetic barriers for defect reactions with the void surface revealed that the correct physical modeling requires the consideration of a reaction boundary condition for defects at the surface, which reveals new void growth dynamics that were not previously considered (see Figure 15). Completing the sharp interface description of the free (void) surface motion due to defect reactions enables the determination of the phase-field model parameters by asymptotic matching. Currently, matching the sharp interface equations to the diffuse interface ones is in process. Due to the complexity of the problem, the diffuse and sharp interface models were initially tested for a single component system; both models are currently being extended to ionic crystals.

In an effort to understand the role of near surface stoichiometry and its dependence on changes in ambient oxygen partial pressure and temperature, a preliminary investigation of defect reactions at $\mathrm{UO}_{2}$ surfaces is being conducted. Figure 16 shows how the stoichiometry at the surface may be affected by the different rates of diffusion and reaction with the surface of anion and cation defects. The highly driven non-equilibrium state of materials under irradiation requires researchers to understand the kinetics of the surface reactions as opposed to the equilibrium situations dealt with in the literature. Therefore, experiments are in progress to investigate the near-surface evolution of the defect state (stoichiometry) in $\mathrm{UO}_{2}$ close to a free surface, and quantum mechanical calculations of energy barriers for defect reactions at the surface. These barriers will be used in transition state theory formulations of the surface reactions, which define the dynamic boundary conditions for the defect concentration fields. This step will ultimately help researchers model stoichiometric changes in irradiated $\mathrm{UO}_{2}$, which are critical in understanding phonon scattering.

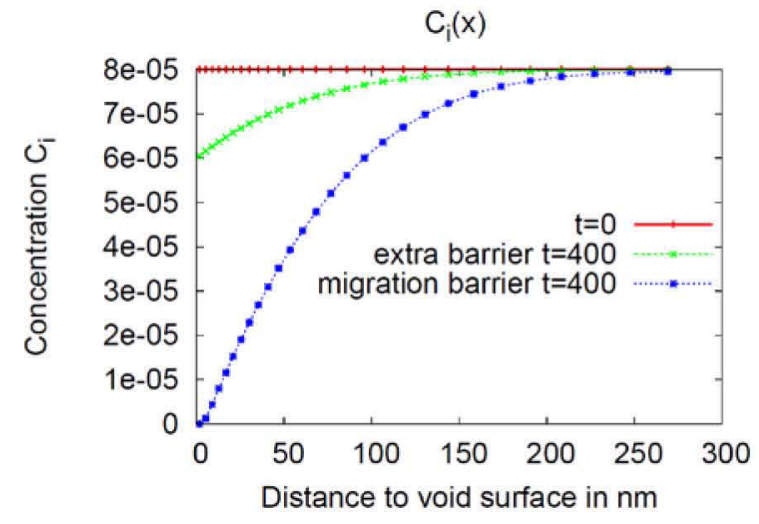

(a)

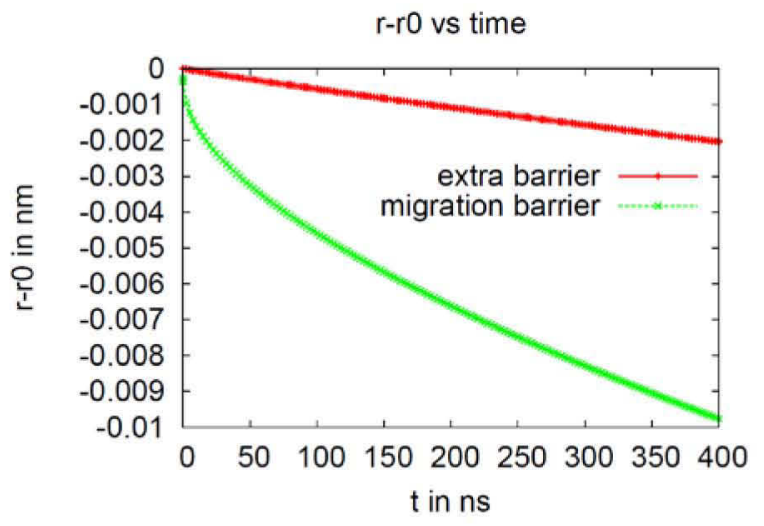

(b)

Figure 15. Influence of an extra barrier for defect surface reactions exceeding the migration barrier by $1 \mathrm{eV}$ on the shrinkage of a 2-dimensional void of radius $100 \mathrm{~nm}$ in copper at $1000 \mathrm{~K}$ with initial interstitial supersaturation of $10^{7}$ : (a) interstitial concentration profile after $400 \mathrm{~ns}$ as a function of distance to the void with and without extra barrier for surface reactions; (b) displacement of the void surface as a function of time with and without extra barrier for surface reactions. 


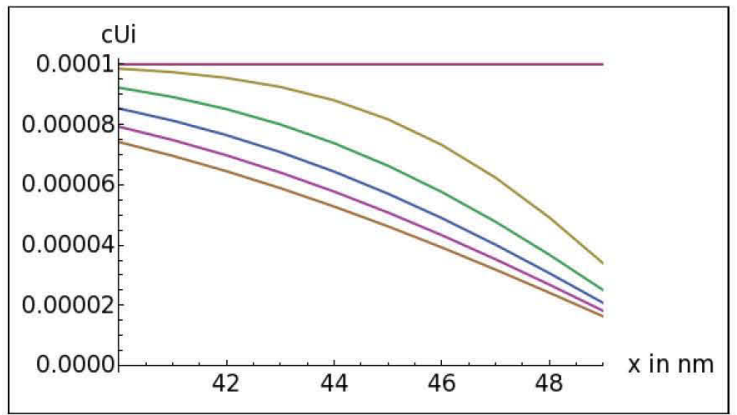

(a)

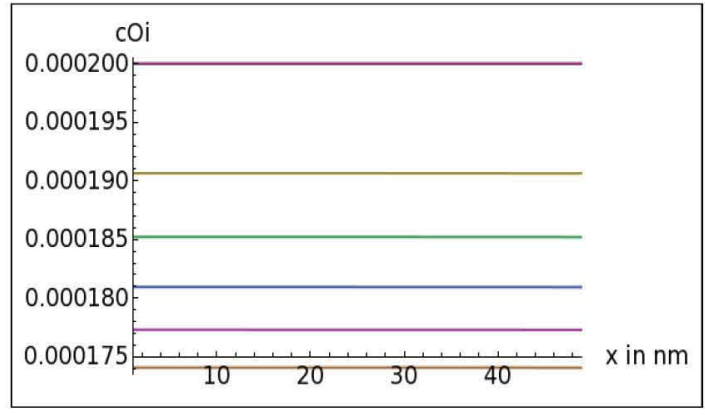

(b)

Figure 16. Uranium (a) and oxygen (b) interstitial concentration profile near a flat surface of an defected but stoichiometric $\mathrm{UO}_{2}$ crystal with initial concentration of uranium interstitials of $\mathrm{c}_{U_{i}}=0.0001$ and, vacancies $\mathrm{Cuv}_{v}=$ 0.01 and according oxygen defects concentrations of $\mathrm{c}_{\mathrm{O}_{i}}=0.0002$ and $\mathrm{cov}_{\mathrm{v}}=\mathbf{0 . 0 1}$. The imbalance of defect fluxes causes changes of stoichiometry near the surface [right end of the figures].

\subsubsection{Atom Probe Characterization}

Quantitative atomic-level 3D compositional analysis was performed on $\mathrm{CeO}_{2}$ pellets irradiated with $300 \mathrm{MeV} \mathrm{Au}{ }^{+}$ions to a fluence of $1 \times 10^{12} \mathrm{~cm}^{-2} \mathrm{t}$ and thin films samples of $\mathrm{CeO}_{2}$ obtained from the Colorado School of Mines that were processed using magnetron sputtering. Samples were run at the Central Analytical Facility at the University of Alabama and Center for Advanced Energy Studies at INL. The facility at the University of Alabama houses the LEAP 3000SI that utilizes a green laser while INL houses the LEAP $4000 \mathrm{hr}$ that utilizes a UV laser.

The objective in the study of ion implanted $\mathrm{CeO}_{2}$ pellets was to characterize the local atomic compositional variations near ion tracks. It was envisioned that ion tracks would be associated with local changes in chemistry that may be able to be identified using atom probe tomography. Samples were run at the University of Alabama and INL. The results of the analysis indicate that although the bulk composition matches closely with complimentary characterization techniques, local stoichiometry changes were observed to be caused by localized heating from the incident laser beam used to ion evaporate the samples during the analysis. Various run parameters were used, with evaporation rate ranging from 0.5 to $3.0 \%$, pulse rate ranging from 50 to $200 \mathrm{kHz}$, temperature ranging from 15 to $90 \mathrm{~K}$, and laser pulse energy ranging from 2.5 to $120 \mathrm{pJ}$. Changing these parameters slightly altered the artifact, but was unable to completely resolve the issue.

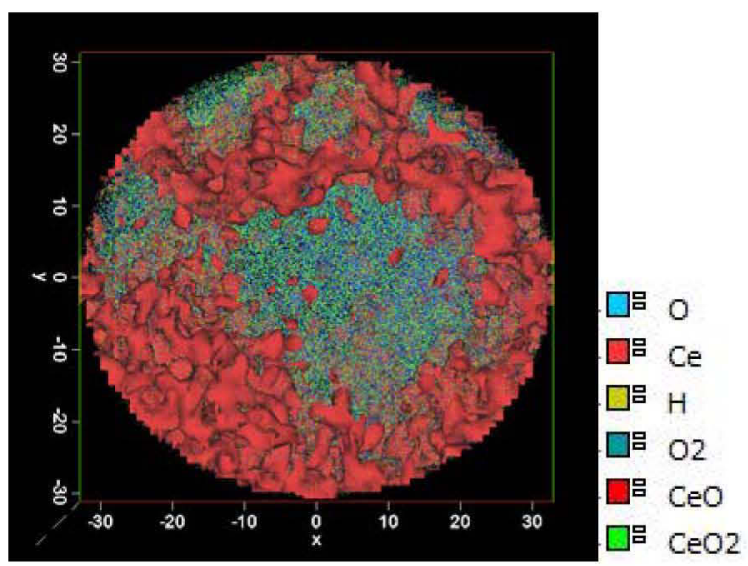

Figure 17. Atom probe reconstruction showing $21 \% \mathrm{Ce}$ iso-concentration surface in red. Enclosed in these surfaces are areas where the Ce concentration is higher than $21 \%$. These concentration gradients did not originate from ion damage but from artifacts caused by laser-assisted evaporation. 
Thin film samples from the Colorado School of Mines were studied to determine the feasibility of studying thin films using atom probe tomography and characterize the atomic level chemistry of magnetron sputtered samples. The thin film samples consisted of a grain size of $100-500 \mathrm{~nm}$ and a thickness of $10 \mu \mathrm{m}$. The samples were run in voltage mode, eliminating the laser artifact displayed on the previous $\mathrm{CeO}_{2}$ pellet samples. An image of the reconstructed tip is shown in Figure 18.

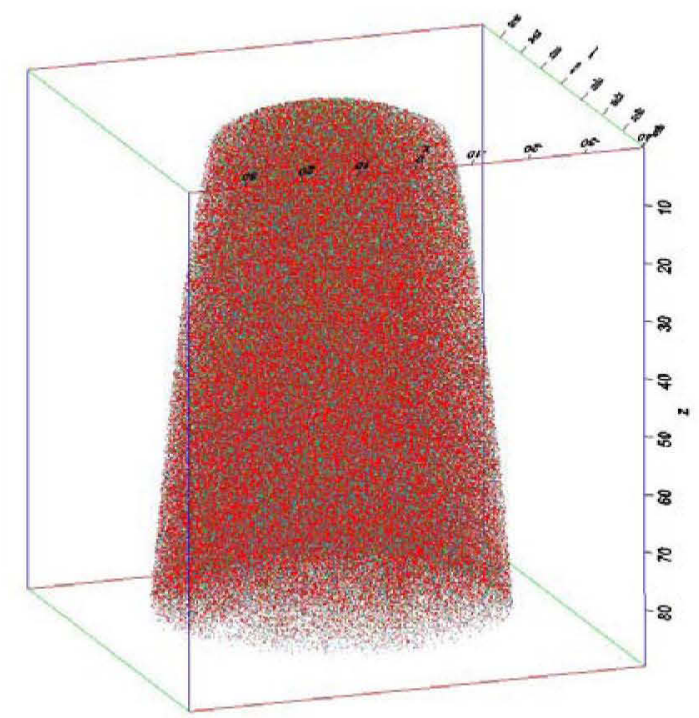

Figure 18. Atom probe reconstruction of thin film $\mathrm{CeO}_{2}$. Red atoms indicate the location of the $\mathrm{Ce}$ atoms while the 0 atoms are colored blue.

The feasibility of analyzing thin film $\mathrm{CeO}_{2}$ was established and it was determined that these samples

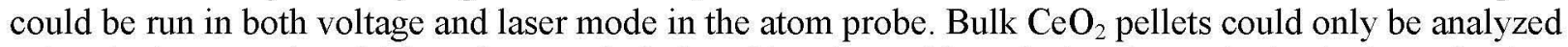
using the laser mode, which unfortunately induced heating artifacts during the analysis. An investigation is currently ongoing to pinpoint the cause of the laser artifacts to avoid this distortion when studying $\mathrm{UO}_{2}$.

\subsection{Conferences and Publications}

\subsubsection{Symposia Organization}

- Anter El-Azab co-organizer a symposium on "Fundamental Science of Defects and Microstructure in Advanced Materials for Energy," MRS Spring 2011, San Francisco, California.

- Anter El-Azab co-organizer a focus session on "Numerical Challenges in Microstructure Modeling for Materials Science," SIAM-CSE11, Reno, Nevada, February 28-March 4, 2011.

- Anter El-Azab co-organizer the Radiation Effects Symposium, $5^{\text {th }}$ International Conference on Multiscale Modeling of Materials, October 4-8, 2010, Freiburg, Germany.

- "Nanoscale Phonon Transport," Fall MRS 2011 (invited Judy Pang and Simon Phillpot).

- David Hurley co-organizer the symposium on Nanoscale Phonon Transport, MRS Fall 2011, Boston, Massachusetts.

- John Moore, co-chairman of several symposia at the International Conference on Metallurgical Coatings and Thin Films. (ICMCTF): 2000 to 2010.

- Six papers to be presented and published at the ICMCTF 2011. Paper related to CSMNF is entitled, "Deposition and Post-annealing of Ceria Films Deposited by Pulsed Unbalanced Magnetron Sputtering," will be an oral presentation at ICMCTF on May 2, 2011. 


\section{Proposed Symposia and Related Activities}

- $\quad$ Radiation Effects in Ceramic Oxide and Novel LWR Fuels Symposium Proposed for the TMS 2012 Conference in Orlando, Florida. Organizers: *Todd Allen, *Jian Gan, Ram Devanathan, Edward Lahoda, ${ }^{*}$ Michele Manuel, Ramprashad Prabhakaran, and *Peng Xu (*indicates affiliation with the INL EFRC).

- Defects and Microstructure Complexity in Materials, for MRS Fall 2012 Meeting in Boston, MA. Organizers: *Anter El-Azab (lead), Fei Gao, Alfredo Caro, and Peter Derlet.

- Anter El-Azab is invited to an Expert Workshop to be held at ORNL in July 2011 on Resolving Long-Standing Modeling Issues for Radiation Damage in Materials.

\subsubsection{Summer School Organization and Sponsorship}

- The 2010 Summer School entitled Atomic-Level Response of Materials to Irradiation was hosted by Los Alamos National Laboratory (LANL) EFRC and co-sponsored by the INL EFRC. This summer school took place at the Inn and Spa at Loretto in Santa Fe, New Mexico during the week of June 2025, 2010. The conference was co-organized by select PIs from LANL, Oak Ridge National Laboratory (ORNL), and INL EFRC: Organizers: *Paul Millet, *Anter El-Azab, Michael Demkowicz, Roger Stoller, Jamie Morris, Bill Weber, and Blas Uberuaga (*indicates affiliation with the INL EFRC).

- Of the multiple sessions that were organized, two talks were given by INL EFRC PIs. One talk entitled "Phase Stability under Irradiation," was presented by Anter El-Azab and another talk entitled "Scattering Techniques for Characterizing Atomic-Scale Radiation," was presented by Ben Larson. The following participants affiliated with INL EFRC were in attendance (Principal Investigators: Dieter Wolf, Anter El-Azab, Ben Larson, and David Hurley; Post-Doctoral Scholars: Thomas Hochrainer, Peng Xu, and Marat Khafizov; Graduate Students: A. R. Hassan, Billy Valderrama, Hunter Henderson, Bowen Deng, and Yangzhong Li.

\section{Proposed Summer School}

- The 2011 Summer School will be hosted and co-sponsored by the INL EFRC. This summer school will take place concurrently with the Advanced Test Reactor (ATR) National Scientific User Facility User's Week 2011 at INL. The event will take place from June 6-10, 2011 and is organized by the following committee: *Todd Allen, *Jian Gan, *Michele Manuel, *Peng Xu, Amit Misra, Michael Demkowicz, Roger Stoller, Jamie Morris, Rod Ewing, Jose Alfredo Caro, and Blas Uberuaga (*indicates affiliation with the INL EFRC).

\subsubsection{Conference Presentations and Posters}

- Gupta, M. Investigation of near Surface Stoichiometry in $\mathrm{CeO}_{2}$, ANS Student Conference, Atlanta, Georgia, April 2011.

- P. Xu, A. Schulte, C. Yablinsky, J. Gan, and T. R. Allen, Characterization of Swift Heavy Ion Damage in $\mathrm{CeO}_{2}, 2011$ TMS Annual Meeting, San Diego, California, February 27, 2011 (poster titled, Grain Growth of $\mathrm{CeO}_{2}$ ).

- C. A.Yablinsky, P. Xu, A.Schulte, H. B. Henderson, B. Valderrama, M. V. Manuel, J. Gan, T. R. Allen, Defect Characterization in Swift Heavy Ion and Proton Irradiated CeO2, 2011 MRS Spring Meeting, San Francisco, California. April 27, 2011.

- M. Khafizov, D. Hurley, Z. Hua, Spatially Resolved Thermal Transport in Nuclear Fuel, INL Research, Design, Development and Demonstration Professional Exchange, Idaho Falls, Idaho, April 14,2011 (poster). 
- A. Schulte, P. Xu, K. Ahmed, A. El-Azab, and T. R. Allen, Grain Growth and Swift Heavy Ion Damage in $\mathrm{CeO}_{2}, 2010$ BES Review Meeting, Rockville, Maryland, September 28, 2010 (poster).

- T. Hochrainer, A. El-Azab and S. Rokkam, Theory and Numerical Simulation of Defect and Microstructure Dynamics in Irradiated Oxides, MMM2010, Freiburg, Germany, October 4-8. 2010 (poster).

- M. Khafizov, D. Hurley, I. Park, J. J. Moore, J. Lin, R. Deskins, A. El-Azab, Thermal Transport in Ceria Thin Films Having Engineered Microstructure, Materials Research Society (MRS) Spring 2011 Meeting, April 25-29, San Francisco, California.

- M. Khafizov, D. Hurley, Measurement of Thermal Transport Using Time-resolved Thermal Wave Microscopy, Materials Research Society (MRS) Spring 2011 Meeting, April 25-29, San Francisco, California.

- S. Rokkam, A. El-Azab and T. Hochrainer, Nonequilibrium Thermodynamics of Void Microstructure and Swelling under Irradiation, Materials Research Society (MRS) Spring 2011 Meeting, April 2529, 2011, San Francisco, California.

- T. Hochrainer, S. Rokkam and A. El-Azab, On the Void-Surface Reaction Kinetics in Irradiated Materials-The Void Growth Problem Revisited, Materials Research Society (MRS) Spring 2011 Meeting, April 25-29, 2011, San Francisco, California.

- T. Hochrainer, A.-R. Hassan and A. El-Azab, Irradiation-Induced Void Microstructure Evolution in Stoichiometric Oxides, Materials Research Society (MRS) Spring 2011 Meeting, April 25-29, 2011, San Francisco, California.

- M. Anitescu. L. C. McInnes, J. Lee, L. Wang, T. Munson, B. Smith, and A. El-Azab, Large-Scale Differential Variational Inequalities for Heterogeneous Materials: An introduction to a new SciDACe Project, CMSNF SIAM-CSE11 Meeting, Reno, Nevada, February 28-March 4, 2011.

- A. El-Azab, Phase Stability Under Irradiation, LANL Summer School, June 20-25, 2011, Santa Fe, New Mexico.

- I-w. Park, J. Lin, J. Moore, M. Manuel, A. El-Azab, T. R. Allen, P. Xu, D. Hurley, and M. Khaflzov, Deposition and Post-annealing of Ceria Films Deposited by Pulsed Unbalanced Magnetron Sputtering, International Conference on Metallurgical Coatings and Thin Films. (ICMCTF), San Diego, California, May 2-6, 2011.

- APS March Meeting 2011, Phonon Dynamics of $\mathrm{UO}_{2}$ at High Temperature.

Invited Presentations:

- Allen, T. "Center for Material Science of Nuclear Fuel," Mechanical Behavior and Radiation Effects of Materials, Contractors Meeting, September 2010.

- Anter El-Azab will give an invited talk entitled "Microstructure Stability in Irradiated Materials," as part of the symposium on Materials Science Challenges for Nuclear Applications, MS\&T Conference, October 16-20, 2010, Columbus, Ohio.

\subsubsection{Conference Attendance}

The following is a list of attendance of principal investigators, post doctoral fellows, and graduate students at conferences, workshops, and the summer school.

\section{Principal Investigators:}

- 2010 LANL EFRC Summer School on the Atomic-Level Response of Materials to Irradiation, Santa Fe, New Mexico (June 20-25, 2010): Dieter Wolf, Anter El-Azab, Ben Larson, and David Hurley. 
- BES Review Meeting, CMSNF Semi-Annual Meeting Fall 2010, CMSNF Semi-Annual Meeting Spring 2011: Todd Allen.

- 5th International Conference on Multiscale Modeling of Materials, October 4-8, 2010, Freiburg, Germany: Anter El-Azab.

- Materials Research Society (MRS) Spring 2011 Meeting, April 25-29, San Francisco, California: Anter El-Azab.

- International Conference on Metallurgical Coatings and Thin Films, April 16-30, 2010, Jianliang Lin.

\section{Post-Doctoral Scholars:}

- 2010 LANL EFRC Summer School on the Atomic-Level Response of Materials to Irradiation, Santa Fe, New Mexico (June 20-25, 2010): Thomas Hochrainer,, Peng Xu, and Marat Khafizov.

- $\quad$ BES Review Meeting, EFRC Summer School 2010, and CMSNF Semi-Annual Meeting Spring 2011: Peng Xu.

- CMSNF Semi-Annual Meeting Spring 2011: Clarissa Yablinsky.

- 5th International Conference on Multiscale Modeling of Materials, October 4-8, 2010, Freiburg, Germany: Thomas Hochrainer.

- Materials Research Society (MRS) Spring 2011 Meeting, April 25-29, San Francisco, California: Thomas Hochrainer and Clarissa Yablinsky.

- International Conference on Metallurgical Coatings and Thin Films, April 27-30, 2010, San Diego, California, In-wook Park.

\section{Graduate Students:}

- 2010 LANL EFRC Summer School on the Atomic-Level Response of Materials to Irradiation, Santa Fe, New Mexico (June 20-25, 2010): Hunter Henderson, Billy Valderrama, A. R. Hassan, Yanzhong $\mathrm{Li}$, and Bowden Deng.

- CMSNF Semi-Annual Meeting Spring 2011: Mahima Gupta.

- ANS Student Conference, Atlanta, Georgia, April 2011: Mahima Gupta.

\subsubsection{Publications}

\section{Peer-reviewed, Archival Journal Publications}

- Hurley, D., Khafizov, M, Shinde, S., "Measurement of Thermal Transport across a Bi Crystal Interface," Journal of Applied Physics, (109, 83504 (2011).

- Hochrainer, T. and A. El-Azab, "Theory of Void Growth in Single Component Systems," in preparation for Physical Review E.

- Aidhy, D. S., D. Wolf, and A. El-Azab, "Comparison of Defect Clustering in Irradiated $\mathrm{CeO}_{2}$ and $\mathrm{UO}_{2}$ from Molecular-Dynamics Simulation," Scripta Materialia, April 2005.

\section{In Preparation: Peer-reviewed, Archival Journal Publications}

- Larson, B and J. Pang, "Thermal Conductivity of $\mathbf{U O}_{2}$ Determined from Lattice Dynamics Using Inelastic Neutron Scattering," in preparation.

- $\quad$ Park, I-w, J. Lin, J. J. Moore, M. Manuel, A. El-Azab, T. Allen, P. Xu, D. Hurley, M. Khaflzov, "Deposition and Post Annealing of Ceria Thin Film Produced by Pulsed DC Magnetron Sputtering," in preparation for Thin Solid Films. 
- $\quad$ Rokkam, S. and A. El-Azab, "Nonequilibrium Thermodynamics of Void Dynamics in Irradiated Materials," in preparation for Physical Review B.

\section{Book Chapter}

- Hurley, D., Shinde, Sullivan, Phonon Transport Across and Along Interfaces, Elsevier.

\subsubsection{Service}

Staff of the EFRC is involved with the following journals:

- John Moore: Editorial board of Surface and Coatings Technology.

- Todd Allen: Editorial board of the Journal of Nuclear Materials and Journal of Nuclear Technology, and editor of Comprehensive Nuclear Materials.

- Jian Gan: Reviewer for the Journal of Nuclear Materials.

- Michele Manuel: Reviewer for Experimental Mechanics, Journal of Metals, Journal of Materials Research, Journal of Alloys and Compounds, Materials Science and Engineering Reviews.

The EFRC Staff is involved in the following committees:

- Curriculum Committee of the Joint EFRC Summer School on Atomic Scale Response to Irradiation, Santa Fe, New Mexico (June 20-25, 2010).

- Anter El-Azab, Michele Manuel, Todd Allen, Peng Xu, Jian Gan.

- $\quad$ TMS Committees

- Nuclear Materials Committee: Todd Allen, Michele Manuel Peng Xu, Clarissa Yablinsky

- Content Development and Dissemination Committee: Michele Manuel

- Integrated Computational Materials Engineering Committee: Michele Manuel

- Young Leaders Committee: Michele Manuel

- Women of TMS Committee: Michele Manuel, Clarissa Yablinsky.

- ANS Materials Science and Technology Division Executive Committee:

- Todd Allen.

- ASM Membership Committee:

- Clarissa Yablinsky.

- International Advisory Board, 5th International Conference on Multiscale Modeling of Materials, October 4-8, 2010, Freiburg, Germany: Anter El-Azab.

- International Organizing Committee, 5th International Conference on Multiscale Modeling of Materials, October 4-8, 2010, Freiburg, Germany.

\section{Staff of the EFRC is involved in the following service activities}

- Lecturer at the Joint EFRC Summer School on Atomic Scale Response to Irradiation, Santa Fe, New Mexico (June 20-25, 2010): Anter El-Azab and Ben Larson.

- Lecturer at the Joint EFRC Summer School at the Advanced Test Reactor (ATR) User Facilities Users Week at INL (June 6-10, 2011): Simon Phillpot, Michele Manuel, Todd Allen, Jian Gan, Peng $\mathrm{Xu}$, Hunter Henderson, Anter El-Azab, Ben Larson.

- Mentoring of REU students: Anter El-Azab mentored Chera Rogers in the summer of 2010 on the research topic, Monte Carlo simulation of diffusion. 
- Participation in the Young Scholar Program at Florida State University: Anter El-Azab mentored two high school students, Nicole Glabinski and Srinivas Balusu during the summer 2010 on the research topic, Monte Carlo simulation of random walk.

- High School Science Research Internship Program: Todd Allen.

- High School Materials Science and Engineering Open House: Michele Manuel.

- Capitol Science and Engineering Fair: Todd Allen.

- Undergraduate design competitions: Michele Manuel.

- Alpha Sigma Mu Honor Society faculty advisor: Michele Manuel.

- Organization of the M.S. and Ph.D. Nuclear Materials Program at CSM: John Moore.

\subsubsection{Technology Transfer, Inventions, and Patents}

None

\subsection{Education and Outreach}

\subsubsection{Education Activities}

Together with the INL's ATR National Scientific User's Facility (NSUF), CMSNF will be coorganizing the 2011 annual summer school on materials, fuels, post irradiation examination, and reactorbased technology development. A joint syllabus committee consisting of members from LANL, ORNL, Massachusetts Institute of Technology (MIT), and CMSNF member institutions developed the curriculum. The school will include 4-1/2 days of courses covering the evolution and impact of microstructural defects on in-reactor material response. In addition, a 1/2-day workshop will be conducted to introduce the capabilities of the ATR NSUF. The school is to be held June 6-10 in Idaho Falls, Idaho. Again, the target audience includes undergraduate and graduate students, post-doctoral researchers, faculty members, and staff scientists.

\subsubsection{Assessment and Evaluation of Education Activities}

During the 2011 INL EFRC Summer School, participants will be involved in a study to critically assess the efficacy of the education objectives of the program. The following has been outlined as the summer school's goals for students and post-doctoral fellows:

Assessment: Provide entrance (upon registration) and exit (upon program completion) surveys.

- The participant's perception of the field of nuclear fuels

- The participant's confidence in their mastery of nuclear science principles

- The participant's confidence in doing research in the nuclear field

- The participant's confidence in working in the nuclear industry.

Evaluation: Impact of the summer school on the knowledge and skills necessary for a researcher in the nuclear field.

- Gains in specific science content (anecdotal)

- Impact of summer school on participants career plans

- Participant's attitude toward the nuclear science discipline

- Participant's attitude toward the nuclear industry 
- Participant's confidence in their ability to be successful in the nuclear field.

The following have been outlined as the summer school's goals for the attending faculty.

Assessment: Provide entrance (upon registration) and exit (upon program completion) surveys.

- The participant's perception of the field of nuclear fuels

- The participant's confidence in teaching nuclear science principles

- The participant's confidence in doing research in the nuclear field

- The participant's confidence in fostering collaborations with others in the nuclear field/industry.

Evaluation: Curricular impact of summer school on the participant's institution.

- Ability to integrate what they learned at the summer school into their classroom

- Impact of summer school on participants career plans

- Participant's attitude toward the nuclear science discipline

- Participant's attitude toward the nuclear industry.

\subsection{Collaborations}

The CMSNF uses facilities outside those of the formal members to accomplish the research and thus, established a number of informal partnerships to augment the work of the center. In cases where research teams are identified with common research goals, the CMSNF finds methods to share research results to the benefit of the center. These collaborations are discussed in the following subsection.

\subsubsection{Joint CMSNF-ANL Effort on Computational Microstructure Simulation}

This effort aims to develop and implement new solution techniques for efficient simulation of void nucleation and growth in irradiated materials. A CMSNF principal investigator (El-Azab) is an unfunded co-principal investigator on an Office of Science project (ASCR) entitled "Large-Scale Differential Variational Inequalities for Heterogeneous Materials." The applied mathematics collaborators at ANL were able to apply this new technique to the void growth model, in collaboration with El-Azab and his students. A Florida State University (FSU) student will be working on this technique at ANL during the summer 2011. This student will be recruited for EFRC in the next fall. A related presentation: M. Anitescu. L. C. McInnes, J. Lee, L. Wang, T. Munson, B. Smith, and A. El-Azab, "Large-Scale Differential Variational Inequalities for Heterogeneous Materials," A SciDAC-e project in support of the CMSNF, SIAM-CSE11 Meeting, Reno, Nevada, February 28-March 4, 2011.

\subsubsection{Scientific Discovery through Advanced Computing Program (SciDAC)}

The Department of Energy's (DOE) Scientific Discovery through Advanced Computing supports multidisciplinary research in a number of scientific areas. The CMSNF and the Towards Optimal Petascale Simulations SciDAC Center for Enabling Technology have established a computational partnership to accelerate efforts to model the microstructural evolution of nuclear fuel. Two related but distinct efforts are being pursued. The project, "Scalable Solvers for Fully Implicit Coupled Nuclear Fuel Modeling," led by Prof. Xiao-Chuan Cai of the Department of Computer Science at the University of Colorado-Boulder and TOPS, is exploring customized algorithms for time integration, linear and nonlinear solvers, and linear and nonlinear preconditioners for the phase field models that are used to model this microstructural evolution. The project, "Large-Scale Differential Variational Inequalities for Heterogeneous Materials," led by Lois Curfman McInnes of the Mathematics and Computer Science Division at ANL and TOPS, is developing an alternative formulation of the governing equations as 
differential variational inequalities along with specialized scalable software for solving this new formulation. Both of these projects will lead to discoveries that will improve the ability of CMSNF staff to solve the complex material science studied in the center.

\subsubsection{University of Illinois}

Nuclear Energy Research Initiative project, "Fundamental Studies of Irradiation-Induced Defect Formation and Fission Product Dynamics in Oxide Fuels:" As part of a DOE-Nuclear Energy funded project, the University of Illinois (Professors Jim Stubbins and Brent Heuser) is studying radiation effects and fission product transport processes in oxide-type nuclear fuels to establish a fundamental understanding of fuel performance. Specifically, researchers are modeling irradiation effects in $\mathrm{CeO}_{2}+\mathrm{x}$, $\mathrm{UO}_{2}+\mathrm{x}$, and $(\mathrm{CeU}) \mathrm{O}_{2}+\mathrm{x}$ surrogate fuels. Additionally, researchers are characterizing thin film $\mathrm{CeO}_{2}$ and $\mathrm{UO}_{2}$ using atom probe tomography. The irradiation effects are induced by ion implantation over a range of energies and doses to simulate the effects of fission product damage. The experimental studies are complimented by modeling using molecular dynamics (MD) simulations of damage cascades in the oxide lattice and kinetic Monte-Carlo (KMC) to study defect dynamics. Because of the common scientific interest, the Illinois researchers have been invited to participate in all CMSNF meetings to ensure no redundancy in effort and to optimize the science performed in each project. A first tangible success of this partnership will be a collaborative presentation at the May 2011 Energy Frontier Research Centers Summit and Forum in Washington, DC.

\subsubsection{Idaho State University}

Nuclear Energy University Program project "Fuel Performance Experiments on the Atomistic Level, Studying Fuel Through Engineered Single Crystal UO 2 :" As part of a DOE-Nuclear Energy funded project, Idaho State University (Professor Eric Burgett) is studying the properties of single crystal $\mathrm{UO}_{2}$. Professor Burgett is establishing a new laboratory in which he will be able to fabricate both single crystal $\mathrm{UO}_{2}$ and $\mathrm{UO}_{2}$ with varying grain sizes. These samples will provide controlled initial microstructures beyond those originally envisioned to be available to the CMSNF, thus greatly improving the scientific discoveries possible. The samples from Professor Burgett will be shared with the CMSNF, in exchange for assistance with advanced characterization techniques (atom probe and transmission electron microscopy) to the benefit of both projects. Samples are anticipated to be available at the end of summer 2011. 


\section{SYNERGISTIC SUPPORT OF PRINCIPAL INVESTIGATORS}

The CMSNF actively uses world-wide user facilities to enhance the research performed.

- ATR NSUF. The ATR NSUF, located at INL, in 2010 has significantly upgraded the infrastructure for performing cutting edge science. Of specific note, in 2010, the ATR NSUF added a local electrode atom probe, a $300 \mathrm{kV}$ field emission gun transmission electron microscope with an energy loss filter, and a focused ion beam system for sample preparation. The CMSNF helped influence the choice of capability added by the NSUF and will actively pursue the advancement of the knowledge of nuclear fuels through the use of this equipment.

- APS at ANL. The CMSNF is actively using the high-energy $x$-ray scattering capability at the APS to understand both short-range and long-range order caused by high-energy ions moving through the fuel lattice.

- GSI Helmholtz Centre for Heavy Ion Research GmbH. The GSI swift heavy ion facility in Germany is providing irradiation services using various ion species with energy greater than $2 \mathrm{GeV}$, which provides a useful tool to understand fission fragment damage, when paired with attenuators such as Al foil.

- The DOE Office of Science SCIDAC-e EFRC Proposal, "Large-Scale Differential Variational Inequalities for Heterogeneous Materials" was recently funded; the Center Investigator Anter El-Azab, FSU is co-principal investigator on this project. He will share a post doctoral associate with ANL staff in Mathematics and Computer Science Division.

- INL's Center for Advanced Modeling and Simulation (CAMS) will provide the research of a post doctoral research assistant (at no cost to the center) to develop new formulations and solvers for the phase-field method, an approach that is central to the center's strategy for modeling microstructure evolution of materials under irradiation. CAMS commitment comes as part of the three-way partnership between the CMSNF EFRC, INL, and the ANL SciDAC-e team.

- Synergistic modeling work is ongoing at the UF (Simon Phillpot) under a DOE Nuclear Energy Research Institute (NERI) (expired in November 2010) and a DOE-Basic Energy Science (BES) CMNS project (expires August 2011) and at FSU under a BES project on mesoscale deformation and two INL-funded projects on microstructural evolution in irradiated materials.

- Synergistic experimental work is ongoing at ORNL in Office of Science funded work on x-ray scattering, x-ray diffraction, and EFRC-funded work on basic defect physics.

- $\quad \mathrm{A} \mathrm{UO}_{2}$ single crystal for the study of phonon lifetimes and anharmonic effects on thermal conductivity properties was provided on loan by Dr. W. J. L. (Bill) Buyers of the National Research Council (NRC) at the Atomic Energy Canada Limited (AECL) in Chalk River, Canada. Dr. Buyers collaborates on the analysis of neutron inelastic-scattering measurements of phonon dispersion and lifetimes (line widths).

- Sintered polycrystalline $\mathrm{UO}_{2}$ pellets for phonon density-of states measurements have been provided by Dr. K.J. McClellan of LANL as facilitated by interactions with INL. Future single crystals of UO2 with specific microstructure such as the inclusion of fission product impurities (e.g., $\mathrm{CeO}_{2}$ alloying) for the investigation of phonon scattering and lifetimes in the presence are projected to be provided by Dr. Eric Burgett of Idaho State University.

- Simon Phillpot and Anter El-Azab are members of the simulation program, and Todd Allen is an advisor within the Consortium for Advanced Simulation of Light Water Reactors (CASL) Energy Innovation Hub; the selection was announced in May 2010. 
- Synergistic work is being performed under a DOE-NE funded, Fuel Cycle Research and Development work package titled, "Laser-based Measurement Technique Development." This work involves development of new characterization tools to provide spatially resolved thermal transport measurements of irradiated nuclear fuel. 


\section{PROGRAM ADMINISTRATION AND PLANNING 3.1 CMSNF Advisory Committee}

The CMSNF established an advisory board consisting of national and international experts in the area of nuclear fuels and held its first meeting in August 2010. The following individuals have agreed to serve on this committee:

- Dr. Vincenzo Rondinella (ITU, Karlsruhe)

- Dr. Marius Stan (ANL)

- Dr. William Weber (ORNL)

- Prof. Sidney Yip (MIT)

- Dr. Rory Kennedy (INL).

The Advisory Committee is chartered with meeting annually to review center activities against seven questions pertaining to execution of strategy:

1. Is the CMSNF organization focused on the correct strategies, objectives, and scientific questions?

2. What activities is the CMSNF doing that it should not be doing?

3. Does the CMSNF have the appropriate staff to successfully implement the CMSNF strategies and objectives?

4. Does the CMSNF have the appropriate facilities to successfully implement the CMSNF strategies and objectives?

5. What additional staff, facilities, or activities would contribute to a more efficient and effective CMNSF outcome and results?

6. How are the CMSNF's recognition, reputation, and eminence viewed?

7. Has the CMSNF targeted the right partners and collaborators?

The findings of the Advisory Committee review conducted in 2010 were published in a report containing the committee's assessment of progress against these seven areas. Recommendations were provided to thrust area leads for incorporation in their research and strategic plans. Highlights included:

- Mission and scientific programs are well defined

- Education and outreach are highly commendable

- The outcomes from the center could have considerable scientific impact as well as technical impact to the nuclear fuels community

- The center has been successful in assembling an excellent team and in setting up a solid, convincing research program

- An effective experimental program has been initiated, and the computational researchers have had excellent results that demonstrate the ability of the phase field method to capture important irradiation effects

- The phonon transport studies are at the forefront of the field and provide excellent information for the benefit of various components of the program. 


\subsection{Staffing}

The staffing plan for the center is based strongly on pairing senior researchers with junior staff (post doctoral associates and graduate students). These pairings are listed in Table 1 below. These junior staffers provide critical day-to-day manpower for the project under the mentorship of the senior investigators; they become leaders in the field of microstructural science and thermal transport.

The EFRC has made good progress filling technical positions. All but one half-time post doctoral positions at ORNL have been filled or are in the process of being filled. ORNL has been successful in managing their staff to cover this shortage. CMSNF has also replaced the first three post docs who transitioned to other positions. Among these new hires were Dr. Jianguo Yu and Dr. David Bai (who will be working on modeling efforts) and Dr. Clarissa Yablinsky at the University of Wisconsin, Madison.

In the summer of 2010, this EFRC suffered the loss of its director. Dave Hill, Deputy Laboratory Director for Science and Technology, appointed Dr. Todd Allen as the interim director. In February, 2011, Dr. Hill appointed Dr. Allen as the permanent director.

Table 1. Staff pairings.

\begin{tabular}{|c|c|c|c|c|c|}
\hline \multicolumn{6}{|c|}{ GRADUATE AND UNDERGRADUATE STUDENTS } \\
\hline & $\begin{array}{l}\text { İ } \\
\text { Ż }\end{array}$ & ב气 & $\frac{\bar{g}}{\frac{\bar{g}}{4}}$ & 苞 & 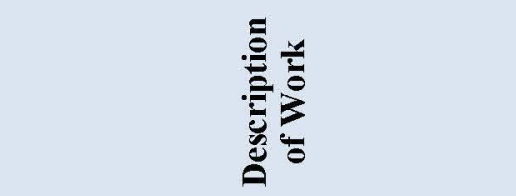 \\
\hline 1 & Yangzhong Li & UF & Simon Phillpot & $\mathrm{Ph} . \mathrm{D}$ & COMB potentials for $\mathrm{U} / \mathrm{UO}_{2}$ \\
\hline 2 & Bowden Den & UF & Simon Phillpot & $\mathrm{Ph} . \mathrm{D}$ & $\begin{array}{l}\text { Thermal transport properties of } \\
\text { dislocations in } \mathrm{UO}_{2}\end{array}$ \\
\hline 3 & Billy Valderrama $^{a}$ & UF & Michele Manuel & $\mathrm{Ph} . \mathrm{D}$ & $\begin{array}{l}\text { Microstructural studies of } \\
\mathrm{CeO}_{2} / \mathrm{UO}_{2} \text { using atom probe }\end{array}$ \\
\hline 4 & Hunter Henderson & UF & Michele Manuel & $\mathrm{Ph} . \mathrm{D}$ & $\begin{array}{l}\text { Microstructural studies of } \\
\mathrm{CeO}_{2} / \mathrm{UO}_{2} \text { using atom probe }\end{array}$ \\
\hline 5 & Anthony Schulte & UW & Todd Allen & Ph.D & Irradiation Stability of $\mathrm{CeO}_{2} / \mathrm{UO}_{2}$ \\
\hline 6 & Walter (Ryan) Deskins & FSU & Anter El-Azab & $\begin{array}{l}\text { M.S. } \\
\text { Ph.D }\end{array}$ & $\begin{array}{l}\text { Monte Carlo solution of Boltzmann } \\
\text { transport equation for phonons }\end{array}$ \\
\hline 7 & A. R. Hassan & FSU & Anter El-Azab & Ph.D & $\begin{array}{l}\text { Stoichiometric changes in } \mathrm{UO}_{2} \\
\text { under irradiation }\end{array}$ \\
\hline 8 & Srujan Rakkam ${ }^{a}$ & FSU & Anter El-Azab & $\mathrm{Ph} . \mathrm{D}$ & $\begin{array}{l}\text { Phase field modeling of void } \\
\text { kinetics in irradiated materials }\end{array}$ \\
\hline 9 & Spencer Morris $^{\mathrm{a}}$ & UW & Todd Allen & B.S. & Irradiation Stability of $\mathrm{CeO}_{2} / \mathrm{UO}_{2}$ \\
\hline 10 & Mahima Gupta $^{\mathrm{a}}$ & UW & Todd Allen & B.S. & Irradiation Stability of $\mathrm{CeO}_{2} / \mathrm{UO}_{2}$ \\
\hline
\end{tabular}


Table 1. (continued).

\begin{tabular}{|c|c|c|c|c|c|}
\hline \multicolumn{6}{|c|}{ POST-DOCTORAL FELLOWS AND RESEARCH ASSOCIATES } \\
\hline & $\underset{\Xi}{E}$ & : & 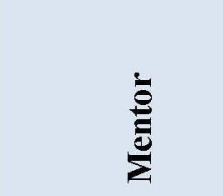 & 莽 & 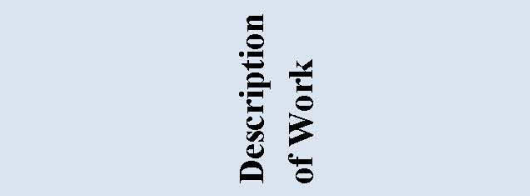 \\
\hline 1 & Jianliang Lin & CSM & John Moore & $\begin{array}{l}\text { Research } \\
\text { Assistant } \\
\text { Professor }\end{array}$ & $\begin{array}{l}\text { Collaborates with professor John } \\
\text { Moore in the Advanced Coatings and } \\
\text { Surface Engineering Laboratory } \\
\text { (ACSSEL) }\end{array}$ \\
\hline 2 & In-wook Park & CSM & John Moore & Post-doc & $\begin{array}{l}\text { Synthesis and characterization of } \\
\mathrm{CeO}_{2} \text { and } \mathrm{UO}_{2} \text { crystalline coatings } \\
\text { using pulsed de magnetron sputtering }\end{array}$ \\
\hline 3 & Peng Xu & UW & Todd Allen & Post-doc & Irradiation Stability of $\mathrm{CeO}_{2} / \mathrm{UO}_{2}$ \\
\hline 4 & Clarissa Yablinsky $^{\mathrm{a}}$ & UW & Todd Allen & Post-doc & Irradiation Stability of $\mathrm{CeO}_{2} / \mathrm{UO}_{2}$ \\
\hline 5 & Thomas Hochrainer & FSU & Anter El-Azab & & $\begin{array}{l}\text { Point defect and microstructure } \\
\text { dynamics in irradiated materials }\end{array}$ \\
\hline
\end{tabular}

In addition to staffing, several principal investigators serve as mentors for students participating in the INL EFRC. The goal of this effort is to increase awareness of cross-institutional efforts while providing the students with opportunities to better understand possible career trajectories. As an example, David Hurley has mentored the following students:

- Zilong Hua - graduate student from Utah State University (Han Ben advisor), thermal conductivity in charged particle irradiated $\mathrm{UO}_{2}$

- Ryan Deskin - graduate student from FSU (Anter El Azab advisor), linking BTE to measurement

Lastly, several students, fellows, and associates have matriculated through the EFRC to find job opportunities in nuclear related fields. Examples are:

- Marat Khafizov transitioned under the advisement of David Hurley from post-doctoral fellow in the INL EFRC to a full-time employee at INL.

- Peng Xu transitioned under the advisement of Todd Allen from post-doctoral fellow in the INL EFRC to a full-time employee at Westinghouse.

The INL EFRC follows a simple strategy of:

- Graduating students

- Assign work that fits the skills and background of the student/fellow/associate

- Help students along and pair them with more senior students or post docs

- Immerse students/fellows/associates completely in the work by involving them in conferences, meeting planning, etc.

- Clearly demonstrate to students/fellows/associates pathways towards successful careers. 


\subsection{Web site and Communications}

\subsubsection{Website}

CMSNF continues to maintain two web-based communities. The public site at http://www.inl.gov/EFRC provides an overview of the center, personnel, and trust areas. The pull-down menu links to various center activities and collaborators. New this year is the addition of an "Education and Outreach" link (which is still under development).

The center has also established an internal, password protected community for collaborating members to share documents, post minutes and presentations from center meetings, and research data and reference documents.

\subsubsection{Monthly Web-based Conference Calls}

CMSNF has established a schedule of monthly conference calls for all team members, including post docs and graduate students. These meetings utilize a technology called GoToMeeting, which is a webbased communication tool that allows the team to give presentations and share technical information from their own desktop. The purpose of these monthly sessions is to communicate/coordinate center activities, share research, and increase collaborations. Each month (on a rotation basis), a principal investigator provides an overview of their research (activities, findings, challenges, and areas where assistance is needed).

\subsubsection{Semi-annual Team Meeting}

Florida State University hosted the semi-annual technical meeting in Tallahassee, Florida, March 911,2011 . The objective of this meeting was to provide a forum for principal investigators, students, and post docs working in the two thrust areas (microstructure under irradiation and thermal transport) to provide an overview of the research being conducted, discuss the path forward (which will be included in the updated roadmap), and provide an opportunity for cross-theme collaboration, and coordination. The center has been operational since August 2009, and the center is now driving toward more concrete outcomes. One of the key outcomes from this meeting was the opportunity for researchers to become better integrated and working toward answering the key research questions.

In addition, two of CMSNF's informal collaborators provided presentations. Both institutions discussed their capabilities and discussed areas of collaboration. The first was a presentation by Idaho State University, a potential supplier of single crystal research samples. The second by the University of Illinois who provided an overview of work they are doing on thin film, single-crystal urania. Day two of the meeting was devoted to strategic planning and discussing areas of cross-theme collaboration and anticipated outcomes and publications for 2012 and 2013. 


\section{FY2011 EFFORTS AND BUDGETS}

\subsection{Summary}

Table 2 shows the overall FY2011 budget distribution for the center by member institution and principal investigator. The university funds are being disbursed via INL subcontracts, whereas the ORNL funding is sent directly to ORNL via a separate FWP.

Table 2. FY2011 budget distribution by institution and principal investigator.

\begin{tabular}{|l|c|}
\hline \multicolumn{1}{|c|}{ Institution (Principal Investigator) } & Funding (\$K) \\
\hline INL modeling (Todd Allen ${ }^{\text {}}$ ) & $220^{\mathrm{a}}$ \\
\hline INL experiments (David Hurley, Jian Gan) & 500 \\
\hline \multicolumn{1}{|c|}{ Total (INL) } & 720 \\
\hline ANL (modeling; Dieter Wolf) & 280 \\
\hline FSU (modeling; Anter El-Azab) & 200 \\
\hline UFL (modeling; SimonPhillpot) & 100 \\
\hline ORNL (experiments; Ben Larson) & 300 \\
\hline UFL (experiments; Michel Manuel) & 100 \\
\hline CSM (experiments; John Moore) & 150 \\
\hline UWM (experiments; Todd Allen ${ }^{b}$ ) & 150 \\
\hline \multicolumn{2}{|c|}{1,280} \\
\hline $\begin{array}{l}\text { a. Includes director's work. } \\
\text { b. Joint appointment with INL }\end{array}$ \\
\hline
\end{tabular}

Within this overall budget framework, the center's technical work is organized around two science themes with an additional focus on education. Because of the importance of the educational mission of the Center, a leadership team was established to ensure proper focus on mentoring, education, and outreach.

Thrust Area 1-Microstructure Development Under Irradiation: The microstructure science under irradiation theme, guided by Anter El-Azab and Todd Allen (discussed in more detail in section 1), aims to develop an experimentally validated, atomistically informed mesoscale model to predict microstructure evolution under irradiation. The highest-level task on developing a unified mesoscale model based on phase-field theory relies on input from the atomic-level simulations on the mechanisms controlling processes, such as point-defect formation and migration under irradiation, void and fission-gas bubble formation, and the phase behavior. Closely coordinated with these multi-scale modeling studies is the experimental characterization, on commensurate length scales, of radiation damage and microstructure evolution on samples produced under carefully controlled synthesis conditions.

Thrust Area 2-Thermal Transport in Irradiated Materials: The theme on thermal transport, guided by Hurley and Phillpot, builds on the underlying microstructure to predict the thermal conductivity under irradiation shown in Figure 4. Phonon scattering processes in irradiated microstructures limit the thermal transport in the presence of irradiation. These simulations are closely coordinated with phonon dispersion and lifetime measurements to be performed at the SNS. The insights thus gained on the unit phonon mechanisms provide the input to the Boltzmann transport- equation prediction of the thermal conductivity, the validity of which is tested via pulsed-laser measurements of thermal diffusivity.

Education: Lead by Michele Manuel and John More, the center has been working to develop a strong internal and external educational component, with specific activities tuned to the development of the 
program and the perceived educational needs at that time. Internally, the center seeks out educational opportunities through which junior staff have an opportunity to increase their knowledge and to which senior staff can contribute as lecturers. Additionally, the center will ensure the post doctoral associates and graduate students are given ample opportunities to assist in report preparation, to present work at conferences, and to present work at team review meetings. The center will also sponsor schools and technical symposia that highlight the Center's work and synergism with other parts of the technical community, including other BES EFRCs.

\subsection{FY2011 Changes in the CMSNF Budget}

None

\subsection{Anticipated FY2011 Carryover}

The estimated carryover funds shown in Table .

Table 3. Estimated carryover into FY2011 (in \$K) by institution and principal investigator.

\begin{tabular}{|l|c|}
\hline \multicolumn{1}{|c|}{ Institution (Principal Investigator) } & Carryover (\$K) \\
\hline INL modeling (Todd Allen ${ }^{\text {a }}$ ) & $312^{\mathrm{a}}$ \\
\hline INL experiments (David Hurley, Jian Gan) & 231 \\
\hline FSU (modeling; Anter El-Azab) & 0 \\
\hline UFL (modeling; Simon Phillpot) & 0 \\
\hline ORNL (experiments; Ben Larson) & 0 \\
\hline UFL (experiments; Michel Manuel) & 0 \\
\hline CSM (experiments; John Moore) & 0 \\
\hline UWM (experiments; Todd Allen ${ }^{b}$ ) & 543 \\
\hline \multicolumn{2}{|c|}{ TOTAL } \\
\hline $\begin{array}{l}\text { a. Includes director's work. } \\
\text { b. Joint appointment with INL }\end{array}$ \\
\hline
\end{tabular}




\section{SCHEDULE STATUS}

In 2010, the key technical and administrative events planned and completed are listed in the following subsection.

\subsection{Technical Achievements and Risks}

A large number of technical accomplishments were achieved by the CMSNF this year and some technical challenges still must be overcome.

- Established a number of informal partnerships to augment the work of the Center (e.g., Idaho State University, University of Illinois)

- Performed and analyzed the first set of heavy ion irradiations at the GSI facility in Germany

- Performed and analyzed the first irradiations using the IVEM facility at Argonne National Laboratory

- Performed density of states measurements at the Spallation Neutron Source $\mathrm{UO}_{2}$ sintered pellets provided by LANL

- Performed phonon dispersion, group velocity and line width measurements at the HFIR reactor using $\mathrm{UO}_{2}$ single crystal sample loaned by Atomic Energy Canada Limited (AECL)

- Completed an analysis of $\mathrm{UO}_{2}$ potentials

- Performed preliminary measurements of thermal transport in $\mathrm{UO}_{2}$ thin films

- Proposed Nuclear Fuel Symposium at 2012 TMS

- Performed and analyzed $\mathrm{CeO}_{2}$ doped with $\mathrm{Nd}$ and $\mathrm{Sm}$ pellets processed by Professor Juan Nino at the University of Florida by atom probe; calculated the degree of solute clustering

- Performed and analyzed commercial $\mathrm{CeO}_{2}$ pellets by atom probe

- Performed and analyzed $\mathrm{CeO}_{2}$ thin films processed by Colorado School of Mines by atom probe

- Performed and analyzed swift ion irradiated $\mathrm{CeO}_{2}$ pellets by atom probe, investigating stoichiometry variations around ion tracks

- Performed first atom probe runs using the 3-D atom probe at the Center for Advanced Energy Studies, associated with INL

- Performed atom probe runs on $\mathrm{CeO}_{2}$ under voltage operating mode and laser operating mode with both green and UV lasers

- Performed and analyze the first irradiations using the Tandem Pelletron accelerator at University of Wisconsin

- Characterized the influence of grain boundaries on phonon mediated thermal transport in $\mathrm{CeO}_{2}$ thin films

- Measured thermal conductivity in sintered $\mathrm{CeO}_{2}$ pellets up to $700^{\circ} \mathrm{C}$

- Established the pulse magnetron sputtering system for depositing $\mathrm{Ce}-\mathrm{O}$ and $\mathrm{UO}$ films

- Changed the stochiometry in $\mathrm{CeO}_{2}$ by using magnetron sputtering process

- Characterized and evaluated the $\mathrm{CeO}_{2}$ films

- Completed training and established conditions for working with depleted uranium magnetron sputtering targets. 


\section{Technical Risks and Challenges}

- Controlling film thickness that must be varied from $50 \mathrm{~nm}$ to $10 \mu \mathrm{m}$ with grain size in the range from $5 \mathrm{~nm}$ to $1.0 \mu \mathrm{m}$ ( $90 \%$ complete) has been challenging: researchers are able to control the two film's size factors (thickness and grain size) by controlling deposition parameters (e.g., target power, deposition temperature, deposition time, post-deposition annealing). However, the grain size cannot be grown perfectly to $1.0 \mu \mathrm{m}$ even if researchers applied the post-deposition annealing process with high temperatures up to $1100^{\circ} \mathrm{C}$. CMSNF researchers may be able to achieve this grain size using substrate heating above $350^{\circ} \mathrm{C}$.

- Determining the energy loss issue in the Monte Carlo thermal transport computations must still be accomplished.

- Determining optimum conditions for atom probe tomography of ceria and eventually urania still must be accomplished to get high-fidelity data.

\subsection{Administrative Achievements and Risks}

A number of administrative accomplishments were achieved by the CMSNF this year and some challenges still must be overcome.

- Completed initial hiring of students, post docs, and technical staff to operate the CMSNF

- Conducted the first formal review of the CMSNF by the external advisory board.

\section{Administrative Risks and Challenges}

- A 50\% post doctoral position at ORNL has not yet been filled as the funding for this post doc needed to be shared by another program. As stated in section 3.2, ORNL has been successful in managing their staff to cover this shortage, but they need to find another willing partner. This search is still in progress. 


\section{REFERENCES}

1. Yin, Q. and S. Y. Savrasov, 2008, "Origin of Low Thermal Conductivity in Nuclear Fuels," Physical Review Letters 100(22): 225504.

2. Li, B. et al., 1999, "Complete Thermal Characterization of Film-on-Substrate System by Modulated Thermoreflectance Microscopy and Multiparameter Fitting," J. Appl. Phys, 86, p. 5314.

3. Callaway J., 1959, "Model for Thermal Conductivity at Low Temperatures," Phys. Rev., 113, p. 1046.

4. Lacroix, D., and Joulain, K., and Lemonnier, D., 2005, "Monte Carlo Transient Phonon Transport in Silicon and Germanium at Nanoscale," Phys. Rev. B, 72, p. 064305.

5. McConnell, A. D., et al., "Thermal Conductivity of Doped Polysilicon Layers," $J$. Microelectromechanical Systems, 10, 360, 2001.

6. Han, Y. J., and Klemens, P. G., 1993, "Anharmonic Thermal Resistivity of Dielectric Crystals at Low Temperatures," Phys. Rev. B, 48, pp. 6033-6042. 